\title{
Direct Proximity Tagging of Small Molecule Protein Targets Using an
}

\author{
Engineered NEDD8 Ligase \\ Zachary B. Hill, ${ }^{1}$ Samuel B. Pollock, ${ }^{1}$ Min Zhuang, ${ }^{1,3}$ and James A. Wells ${ }^{1,2^{*}}$ \\ ${ }^{l}$ Department of Pharmaceutical Chemistry, ${ }^{2}$ Department of Cellular and Molecular Pharmacology, University of California, San \\ Francisco, California, USA. ${ }^{3}$ School of Life Sciences and Technology, ShanghaiTech University, Shanghai, China.
}

Contents

I. Supporting Information Figures 1-10

II. Synthesis of DS1 and MS1

A. General Information

B. Synthetic Scheme for DS1

C. Synthetic Scheme for MS1

III. Protein Expression and Purification

A. Expression of His-Biotin-NEDD8

B. Protein Kinase Expression

C. Bcl-xL Expression and Purification

D. Overlap Extension PCR Method for Generating NEDDylator Fusion Proteins

E. Site-Directed Mutagenesis to Generate NEDDylator(10 $10 \mathrm{~K})$

F. PCR Method for Generating NEDDylator(7AK)-BAD

G. NEDDylator Expression and Purification

H. Preparation and Purification of NEDDylator-Small Molecule Conjugates

IV. In Vitro Activity Assays

V. NEDDylation Assays

A. Assay Conditions for NEDDylation of Recombinant Protein

B. Assay Conditions for NEDDylation of Cell Lysates

C. NEDDylation and Affinity Capture of NEDDylated Proteins from SILAC A549 cell lysates

D. Mass Spectrometry and Data Analysis

VI. Supplemental References 


\section{Supporting Information Figures 1-10}

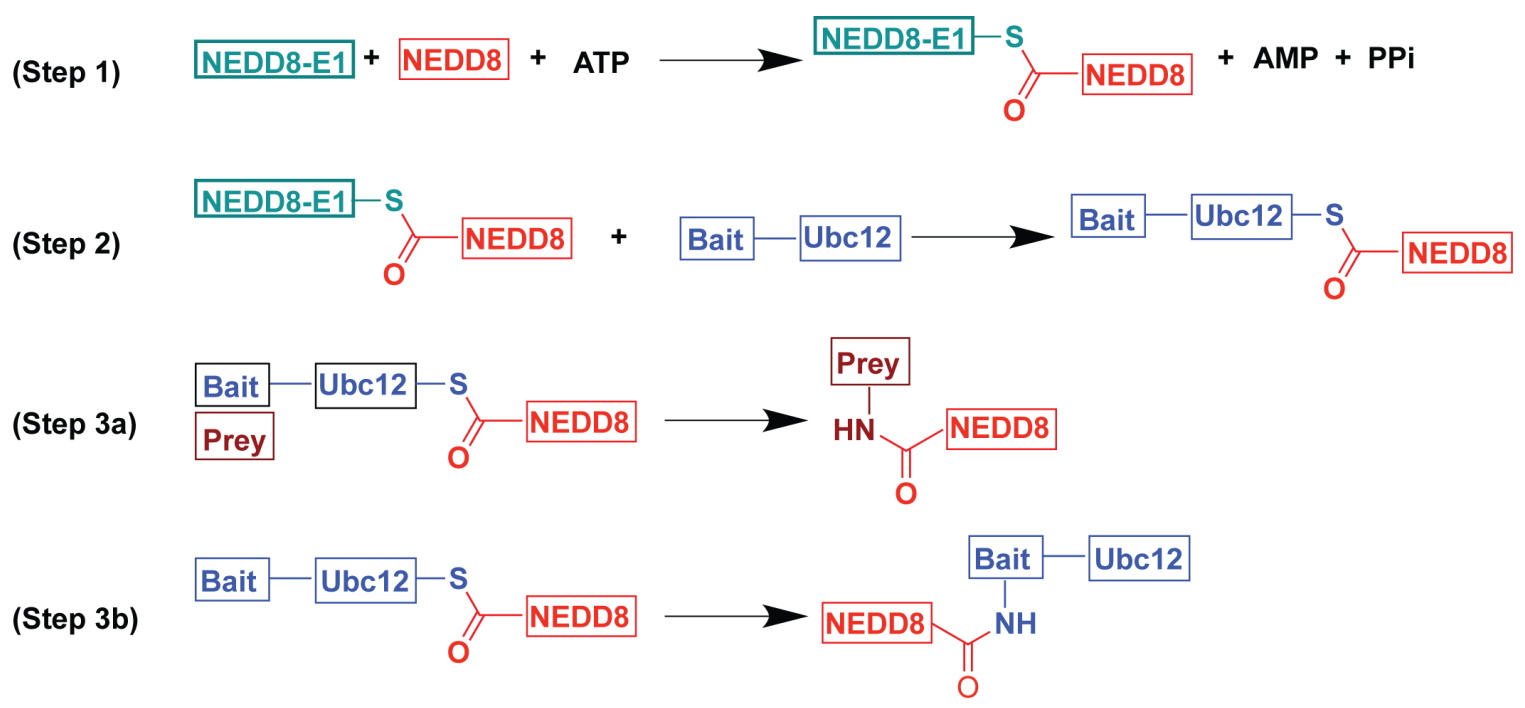

Figure S1. Mechanistic diagram of the NEDDylator enzyme cascade. Step 1 is the ATP dependent formation of a thioester bond between the $C$-terminal carboxylate of NEDD8 and the active site cysteine of NEDD8-E1. Step 2 is a transthioesterfication of NEDD8 from the active site of the E1 to the active site of the NEDD8-E2, Ubc12. Step 3 is the transfer of NEDD8 onto the $\varepsilon$-amine of a surface lysine on either a prey protein (trans-NEDDylation) as depicted in step $3 a$ or the NEDDylator itself (self-NEDDylation), depicted in step $3 b$.

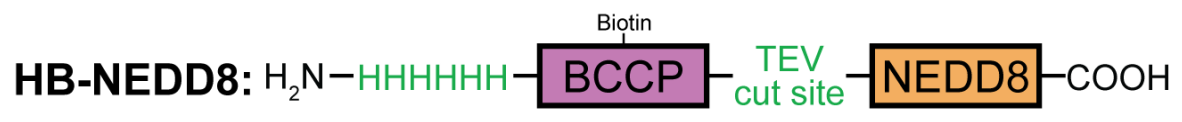

Figure S2. Linear representation of the $\mathrm{His}_{6}$ and biotin tagged version of the ubiquitin-like protein, NEDD8, used in these studies. BCCP = biotin carboxyl carrier protein. HB-NEDD8 has a molecular weight of $\sim 20.7 \mathrm{kDa}$. 


\begin{tabular}{c|c|c|c|c|c}
\cline { 2 - 6 } & \multicolumn{2}{c}{ ABL-3D } & \multicolumn{2}{c}{ SRC-KD } & SRC-KD(T338I) \\
& Dasatinib & DS1 & Dasatinib & DS1 & DS1 \\
\hline Free Inhibitor & $<10$ & $15 \pm 1$ & $<1$ & $<1$ & $>30,000$ \\
\hline NEDDylator(S4) & N/A & $50 \pm 5$ & N/A & $8 \pm 2$ & N/T \\
\hline NEDDylator(S10) & N/A & $95 \pm 4$ & N/A & $14 \pm 2$ & N/T
\end{tabular}

Figure S3. (a) In vitro $\mathrm{IC}_{50}$ values of dasatinib, DS1, NEDDylator(S4)-DS1 and NEDDylator(S10)-DS1, for ABL-3D, SRC-KD, and SRC-KD(T338I). The IC $_{50}$ values measured for dasatinib were below the limit of detection for our assay but dasatinib has previously been reported to have a $K_{d}$ of $0.53 \mathrm{nM}$ for $\mathrm{ABL}$ and $0.21 \mathrm{nM}$ for SRC. ${ }^{1}$ All assays were performed in quadruplicate. $\mathrm{N} / \mathrm{T}=$ not tested, N/A = not applicable.

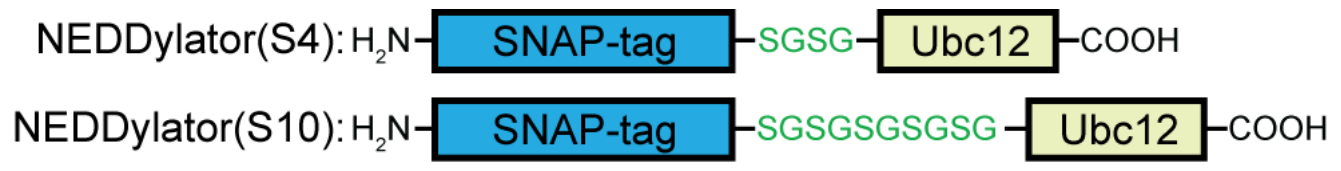

Figure S4. Linear diagram of the SNAP-tag-Ubc12 fusion proteins generated for this study.

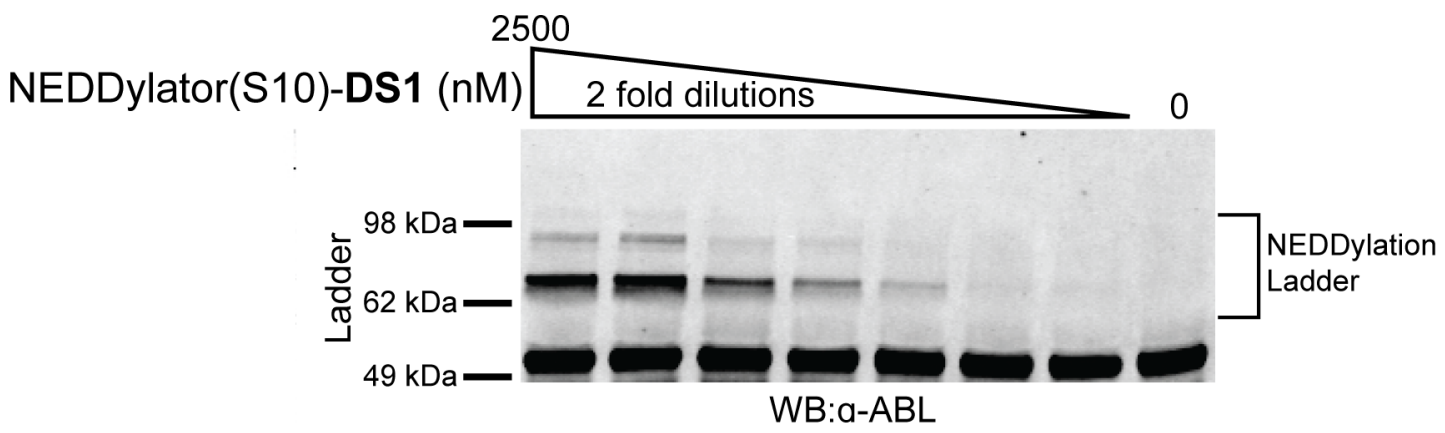

Figure S5. Western blot showing dose dependent NEDDylation of ABL-3D by NEDDylator(S10)-DS1. 


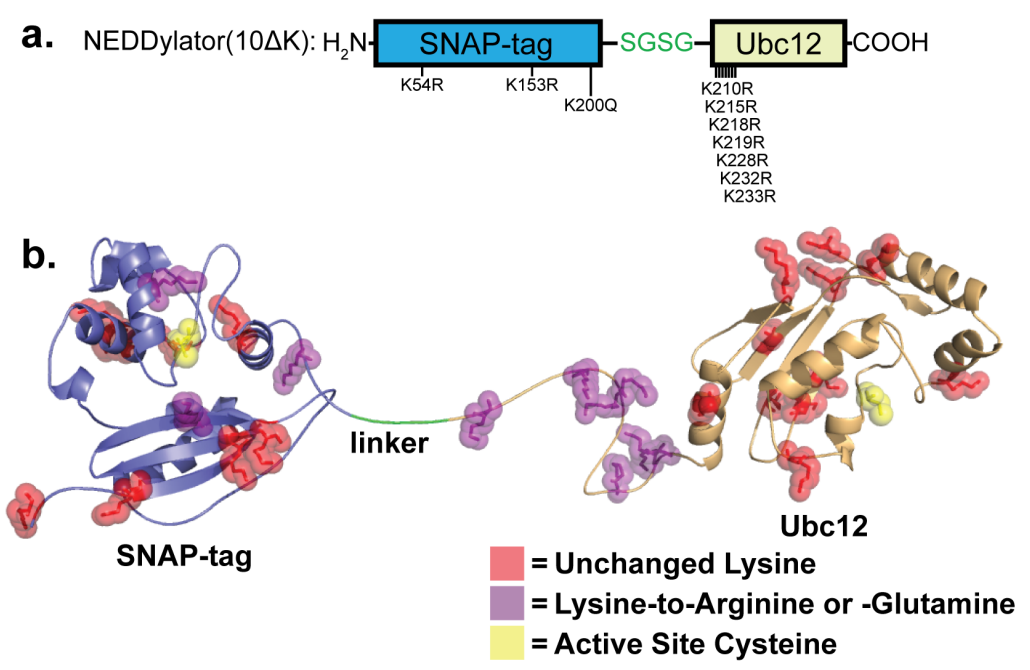

c.

d.

e.

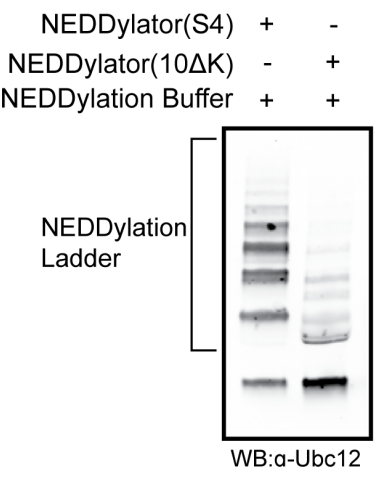

NEDDylator(S4)-DS1 + -

NEDDylator(10AK)-DS1 - +

NEDDylation Buffer $+\quad+$

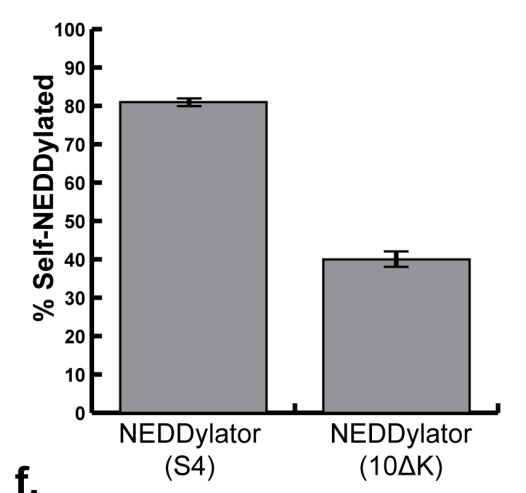

f.

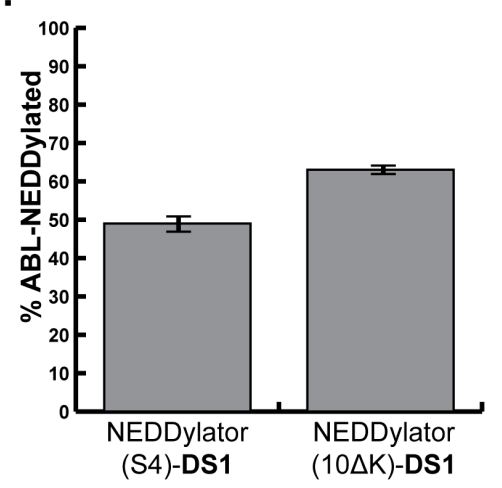

Figure S6. A 10-lysine-to-arginine/glutamine mutant of NEDDylator(S4), NEDDylator(10 $\Delta \mathrm{K})$, shows reduced self-NEDDylation and improved trans-NEDDylation of ABL-3D (a) A linear diagram of NEDDylator $(10 \Delta \mathrm{K})$ depicting the sites of mutations introduced to reduce selfNEDDylation. (b) A cartoon model of NEDDylator $(10 \Delta \mathrm{K})$ showing the locations of mutated and unchanged side chains of lysines as well as active site cysteines. (c) A representative Western blot showing the reduced self-NEDDylation of NEDDylator(10 $\Delta \mathrm{K})$ relative to NEDDylator(S4). (d) Percent self-NEDDylation of NEDDylator(S4) and NEDDylator(10 $\Delta K$ ) based on Westernblotting for $\alpha$-Ubc12. Error bars represent standard error of the mean, $n=3$. (e) A representative Western blot showing the increased trans-NEDDylation of ABL-3D by NEDDylator(10 $\Delta \mathrm{K})$-DS1 relative to NEDDylator(S4)-DS1. (f) Percent trans-NEDDylation of ABL-3D by 


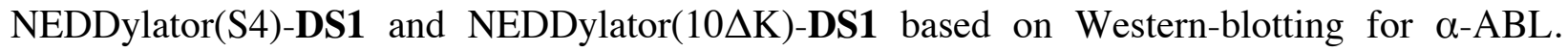
Error bars represent standard error of the mean, $\mathrm{n}=3$

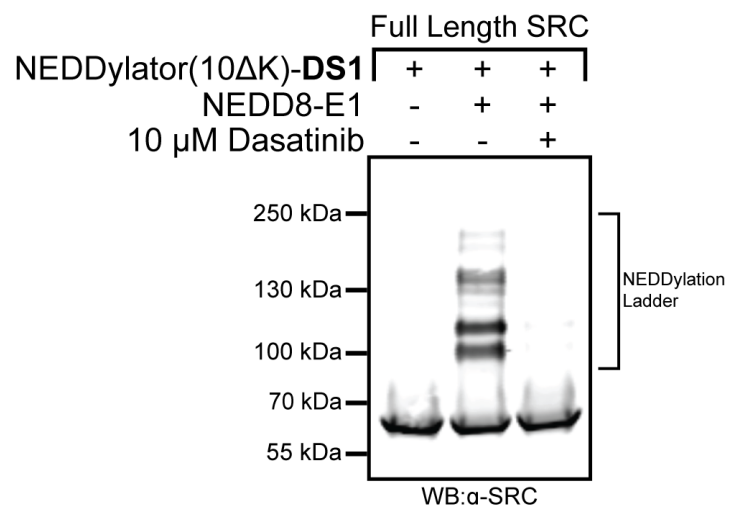

Figure S7. NEDDylator(10 $\Delta$ K)-DS1 labels full-length SRC in a NEDD8-E1 and dasatinibdependent manner.

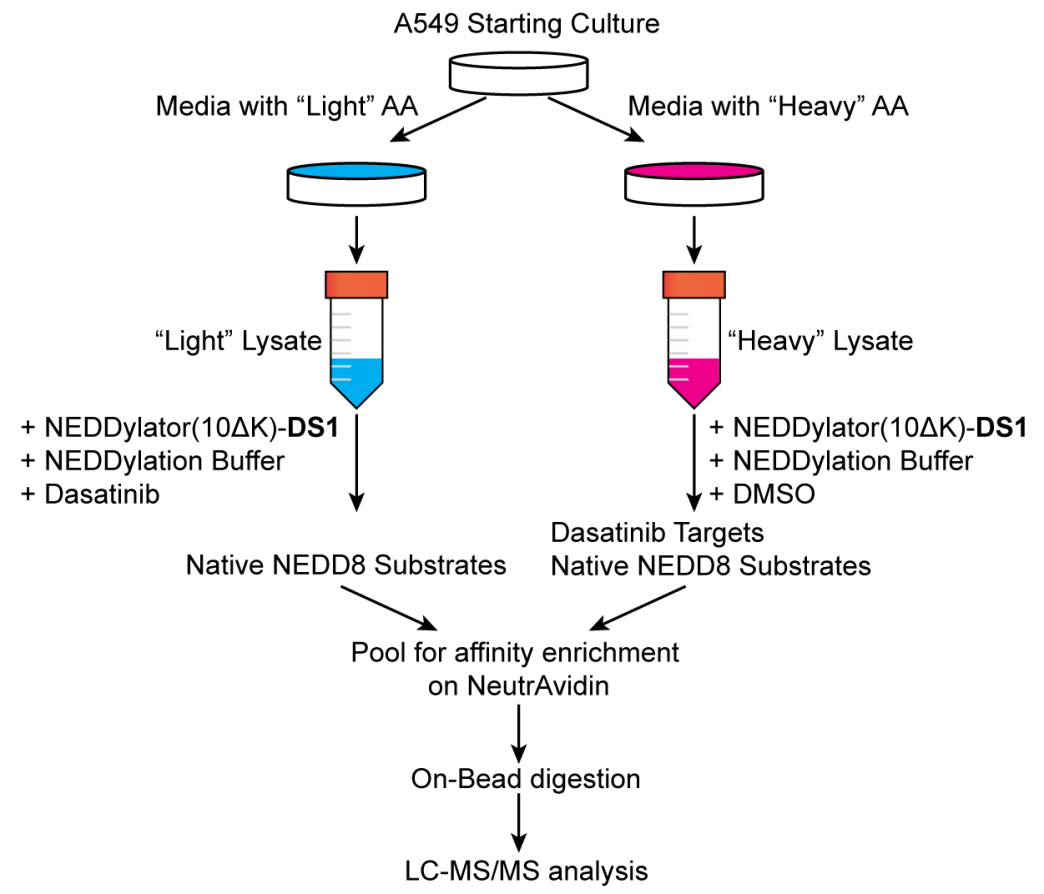

Figure S8. Schematic of the workflow for the SILAC-based NEDDylation, enrichment, and identification experiment performed in this study. 


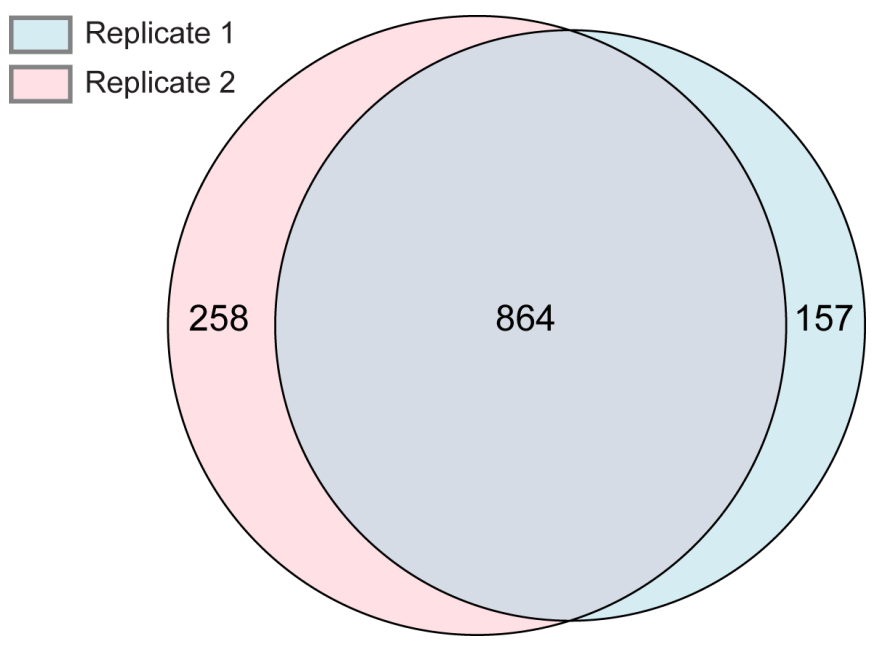

Figure S9. Venn Diagram of the number of proteins identified for two replicate SILAC experiments described in Figure S8.

a.

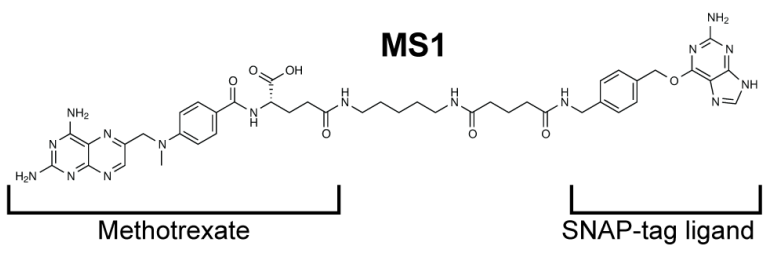

C.

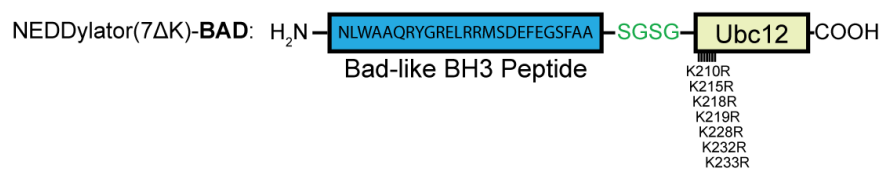

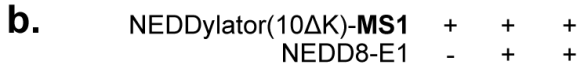

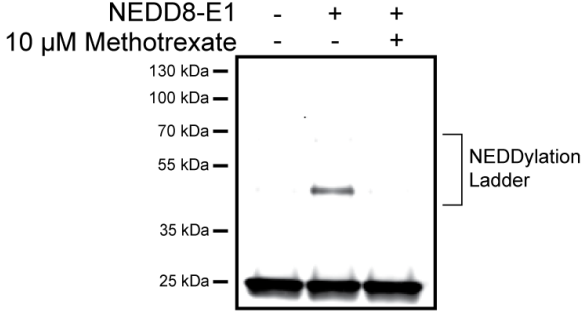

WB:a-DHFR

d.

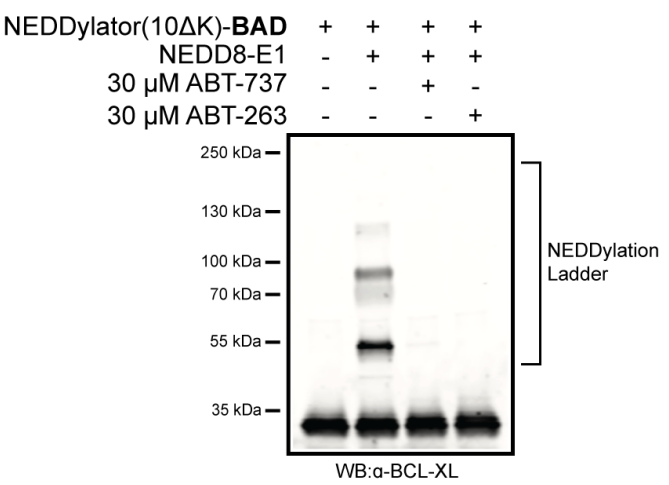

Figure S10. The NEDDylator is capable of tagging protein targets beyond kinases, displaying cognate ligands. (a) MS1, a derivative of methotrexate capable of being displayed from

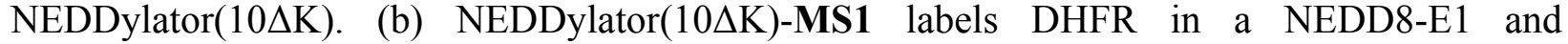
methotrexate-dependent manner. (c) NEDDylator(7 $\Delta \mathrm{K})$-BAD, a NEDDylator construct displaying a Bcl-xL-binding Bad-like BH3 peptide as a genetic fusion at its $N$-terminus. (d) NEDDylator(7 $\Delta \mathrm{K})$-BAD labels Bcl-xL in a NEDD8-E1 and BAD-like-peptide-dependent manner. Pre-incubation with the known BAD-like BH3 mimetic small molecules ABT-737 and ABT-263 inhibits NEDDylation of Bcl-xL by NEDDylator(7 $\Delta \mathrm{K})$-BAD. 


\section{Synthesis of DS1 and MS1}

\section{A. General Information}

Unless otherwise noted, all reagents were obtained from commercial suppliers and used without purification. ${ }^{1} \mathrm{H}-\mathrm{NMR}$ spectra were obtained on a Varian $400 \mathrm{MHz}$ instrument at room temperature. Chemical shifts are reported in ppm, and coupling constants are reported in Hz. All splitting patterns reported are the apparent splitting. Mass spectrometry was performed on a Waters Micromass ZQ.

General HPLC Purification Conditions: Preparatory reverse-phase $\mathrm{C}_{18}$ column $(50 \mathrm{x} 19 \mathrm{~mm})$, Methanol/Water-0.05\% formic acid gradient: 5:95 to $100: 0$ over $30 \mathrm{~min} ; 20 \mathrm{~mL} / \mathrm{min} ; 254 \mathrm{~nm}$ detection for $35 \mathrm{~min}$.

General Analytical HPLC Conditions: $C_{18}$ column $(50$ x $4.6 \mathrm{~mm})$, Methanol/Water-0.1\% formic acid gradient: 5:95 to 100:0 over $8 \mathrm{~min}$ or $13 \mathrm{~min}$ : Flow rate $=1 \mathrm{~mL} / \mathrm{min}$; ELSD and 254 $\mathrm{nm}$ detection for 10 or $15 \mathrm{~min}$.

\section{B. Synthetic Scheme for DS1}
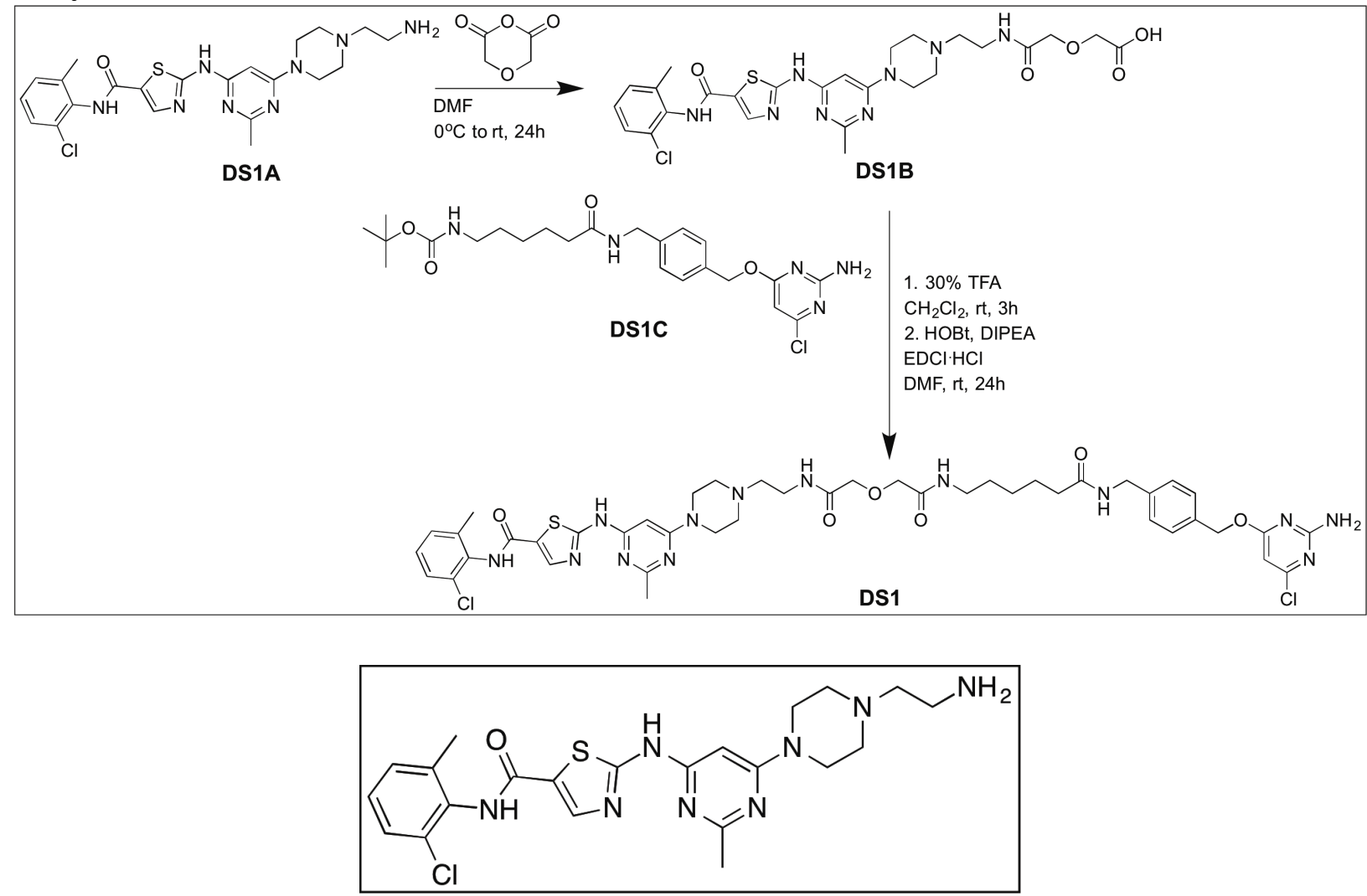

[DS1A] was prepared using a previously published procedure. ${ }^{2}$ 


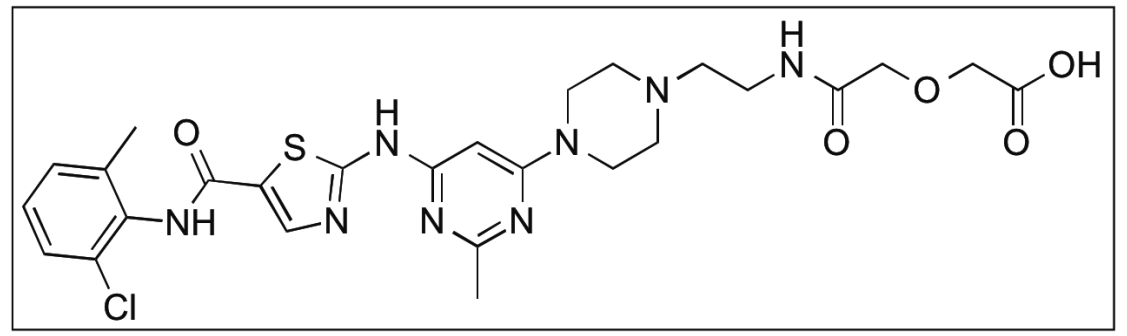

[DS1B] Diglycolic Anhydride (7.2 mg, $61.6 \mu \mathrm{mol}, 1.2$ equiv) as added to a solution of [DS1A] (35 mg, $51.3 \mu \mathrm{mol}, 1.0$ equiv) in DMF $(260 \mu \mathrm{L})$ stirring in an ice bath. The reaction was allowed to warm to room temperature and was stirred for $24 \mathrm{hr}$ at which time the reaction was concentrated in vacuo. The product was used in the next step with no further purification. Calcd for $\mathrm{C}_{26} \mathrm{H}_{32} \mathrm{ClN}_{8} \mathrm{O}_{5} \mathrm{~S}\left(\mathrm{M}+\mathrm{H}^{+}\right)$: 603.19; Found 603.2.

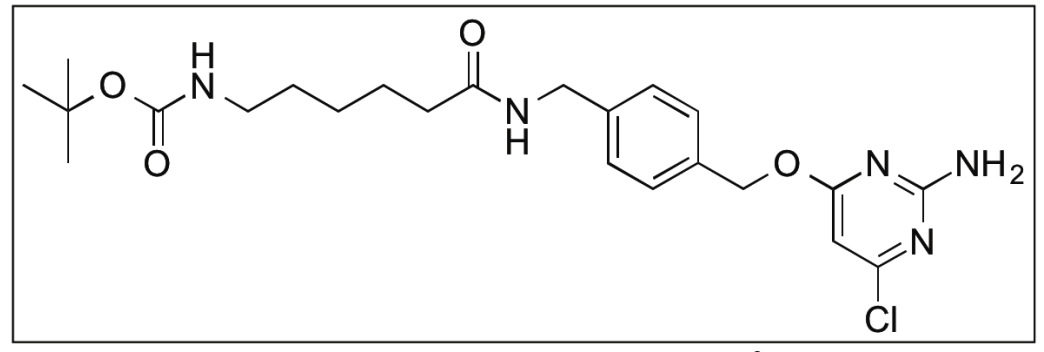

[DS1C] was prepared using a previously published procedure. ${ }^{3}$

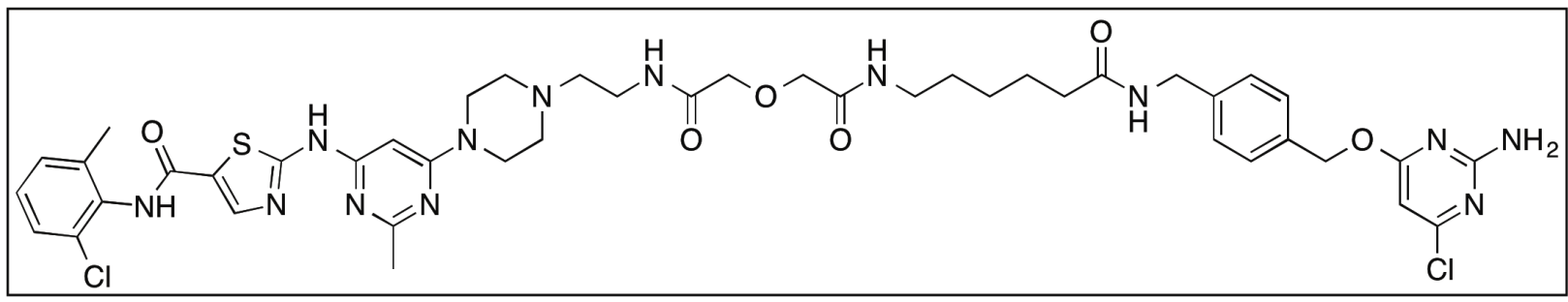

[DS1] A solution of [DS1C] (13.5 mg, $28 \mu \mathrm{mol}, 1.3$ equiv) dissolved in 30\% TFA/DCM (220 $\mu \mathrm{L})$ was stirred at room temperature for $3 \mathrm{hr}$. Toluene $(1 \mathrm{~mL})$ was added and the reaction was concentrated in vacuo. This was repeated 2 additional times. The product was used in the next step without further purification.

To a mixture of deprotected [DS1C] ( $28 \mu \mathrm{mol}, 1.3$ equiv), [DS1B] (12.6 mg, $21 \mu \mathrm{mol}, 1$ equiv), $\mathrm{HOBt} \cdot \mathrm{H}_{2} \mathrm{O}(4.3 \mathrm{mg}, 28 \mu \mathrm{mol}, 1.3$ equiv), and DIPEA (11 $\mu \mathrm{L}, 64 \mu \mathrm{mol}, 3$ equiv) in DMF $(105 \mu \mathrm{L}), \mathrm{EDCI} \cdot \mathrm{HCl}(5.4 \mathrm{mg}, 28 \mu \mathrm{mol}, 1.3$ equiv) was added. The reaction was stirred at room temperature for $24 \mathrm{~h}$, at which time the reaction was dissolved in DMSO and purified using General HPLC Purification Conditions to obtain $2.4 \mathrm{mg}$ of pure [DS1] (12\% yield). ${ }^{1} \mathrm{H}$ NMR (400 MHz, Methanol-d $\left.d_{4}\right) \delta 8.19(\mathrm{~s}, 1 \mathrm{H}), 7.42-7.35(\mathrm{~m}, 3 \mathrm{H}), 7.32(\mathrm{~m}, 4 \mathrm{H}), 6.15(\mathrm{~s}, 1 \mathrm{H}), 6.11(\mathrm{~s}$, $1 \mathrm{H}), 5.34(\mathrm{~s}, 2 \mathrm{H}), 4.38(\mathrm{~s}, 2 \mathrm{H}), 4.11(\mathrm{~d}, J=6.4 \mathrm{~Hz}, 4 \mathrm{H}), 3.71(\mathrm{t}, J=8 \mathrm{~Hz}, 2 \mathrm{H}), 3.40-3.22(\mathrm{~m}$, $12 \mathrm{H}), 2.53(\mathrm{~s}, 3 \mathrm{H}), 2.35(\mathrm{~s}, 3 \mathrm{H}), 2.27(\mathrm{t}, J=7.3 \mathrm{~Hz}, 2 \mathrm{H}), 1.72-1.63(\mathrm{~m}, 2 \mathrm{H}), 1.62-1.52(\mathrm{q}, J=7.3$ $\mathrm{Hz}, 2 \mathrm{H}), 1.43-1.33(\mathrm{~m}, 2 \mathrm{H})$. Calcd for $\mathrm{C}_{44} \mathrm{H}_{54} \mathrm{Cl}_{2} \mathrm{~N}_{13} \mathrm{O}_{6} \mathrm{~S}\left(\mathrm{M}+\mathrm{H}^{+}\right)$: 962.34; Found 962.3. 

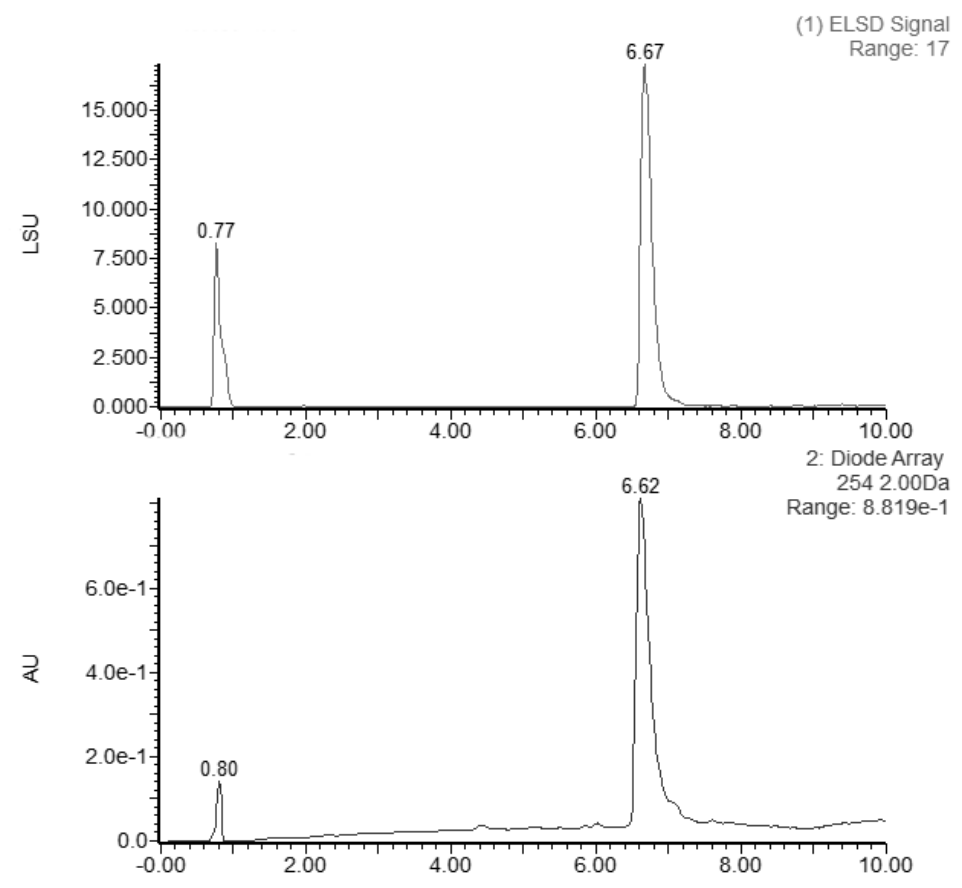

Figure S11. Analytical HPLC trace of DS1 using the general analytical HPLC conditions. The top panel is the signal from the light-scattering detector (ELSD). The bottom panel is absorbance at $254 \mathrm{~nm}$.

\section{Synthetic Scheme for MS1}

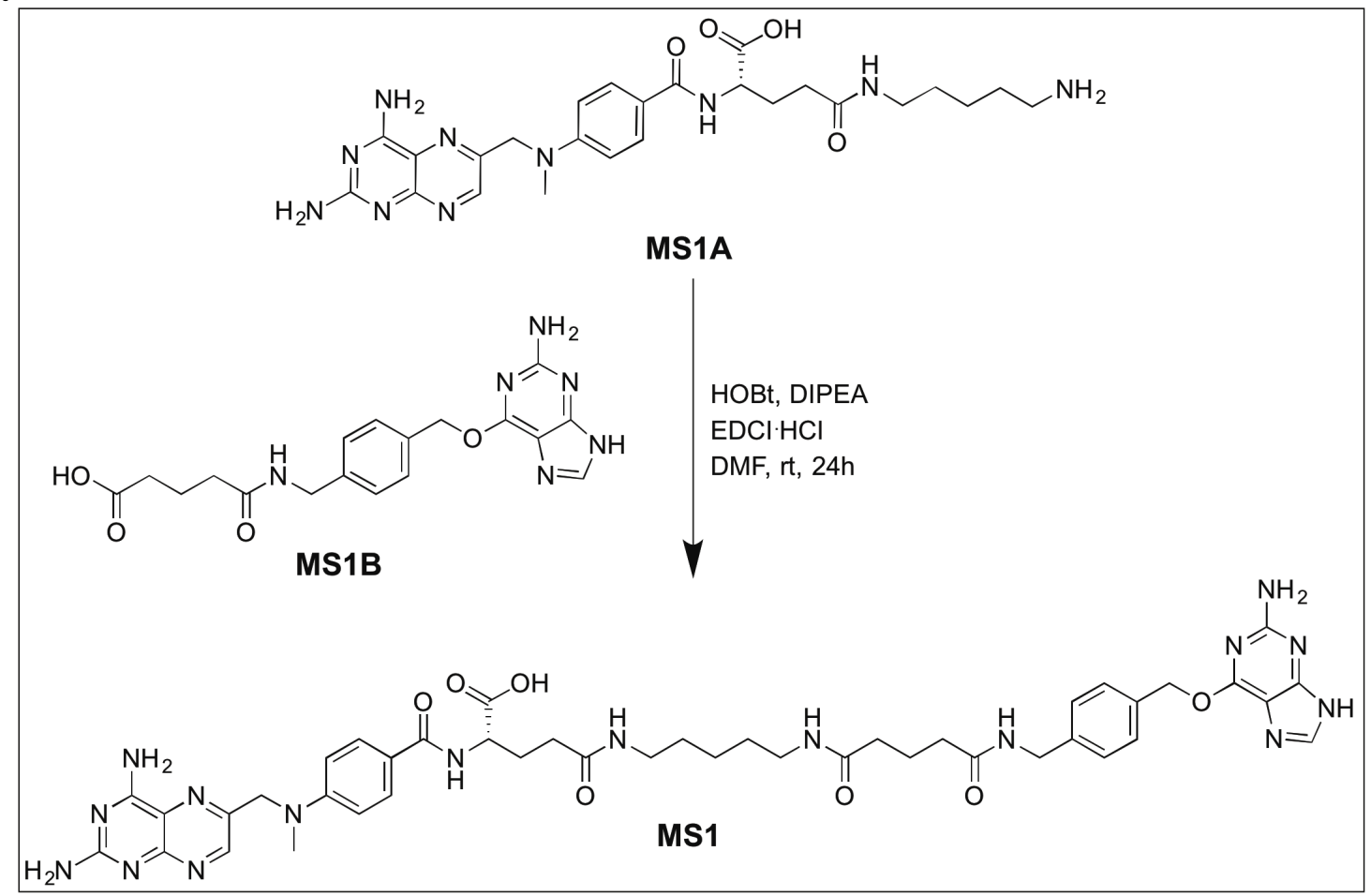




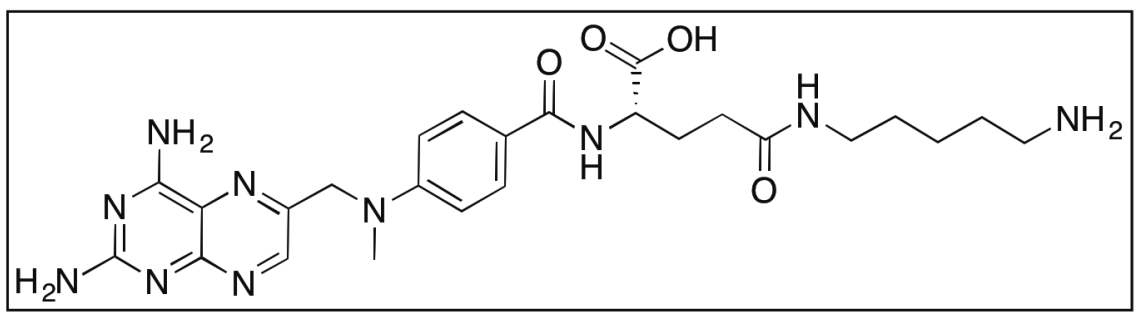

[MS1A] was purchased from Carbosynth and used without further purification.

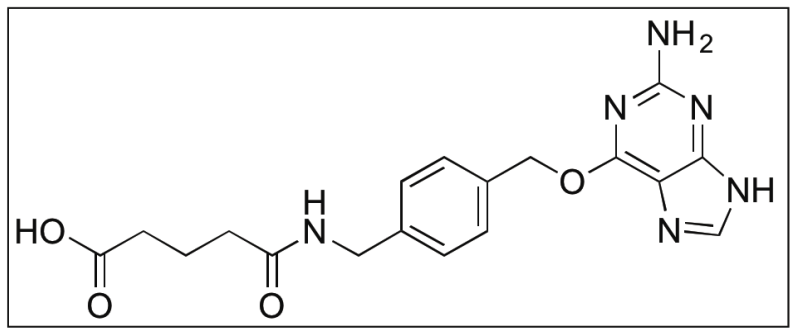

[MS1B] was prepared using a previously published procedure. ${ }^{4}$

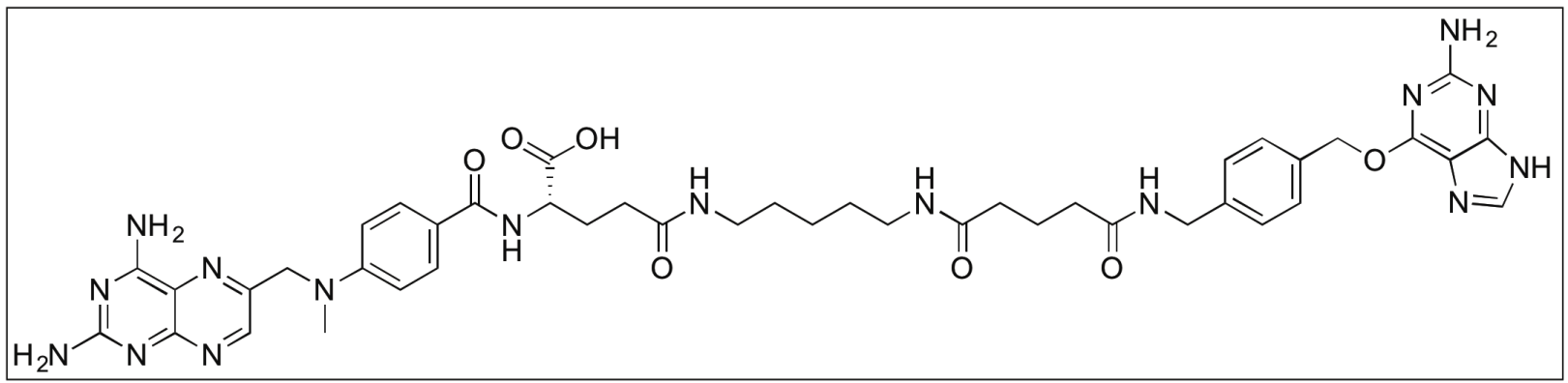

[MS1] To a mixture of [MS1A] $(9 \mathrm{mg}, 17 \mu \mathrm{mol}, 1$ equiv), [MS1B] $(8.4 \mathrm{mg}, 22 \mu \mathrm{mol}, 1.3$ equiv), $\mathrm{HOBt} \cdot \mathrm{H}_{2} \mathrm{O}(3.4 \mathrm{mg}, 22 \mu \mathrm{mol}, 1.3$ equiv), and DIPEA ( $8.9 \mu \mathrm{L}, 51 \mu \mathrm{mol}, 3$ equiv) in DMF $(85 \mu \mathrm{L}), \mathrm{EDCI} \cdot \mathrm{HCl}(4.2 \mathrm{mg}, 22 \mu \mathrm{mol}, 1.3$ equiv) was added. The reaction was stirred at room temperature for $24 \mathrm{~h}$, at which time the reaction was dissolved in DMSO and purified using General HPLC Purification Conditions to obtain $0.9 \mathrm{mg}$ of pure [MS1] (5.8\% yield). ${ }^{1} \mathrm{H} \mathrm{NMR}$ $\left(400 \mathrm{MHz}\right.$, Methanol- $\left.d_{4}\right) \delta 8.57(\mathrm{~s}, 1 \mathrm{H}), 7.83(\mathrm{~s}, 1 \mathrm{H}), 7.74(\mathrm{~d}, J=8.8 \mathrm{~Hz}, 2 \mathrm{H}), 7.48-7.43(\mathrm{~m}$, 2H), 7.31-7.25 (m, 2H), $6.84(\mathrm{~d}, J=8.8 \mathrm{~Hz}, 2 \mathrm{H}), 5.52(\mathrm{~s}, 2 \mathrm{H}), 4.93(\mathrm{~s}, 2 \mathrm{H}), 4.37-4.33(\mathrm{~m}, 3 \mathrm{H})$, $3.23(\mathrm{~s}, 3 \mathrm{H}), 2.69$ (t, $J=6.6 \mathrm{~Hz}, 2 \mathrm{H}), 2.29-2.16(\mathrm{~m}, 8 \mathrm{H}), 1.97-1.86$ (m, 4H), 1.47-1.39 (m, 4H), 1.30-1.24 (m, 2H). Calcd for $\mathrm{C}_{43} \mathrm{H}_{53} \mathrm{~N}_{16} \mathrm{O}_{7}\left(\mathrm{M}+\mathrm{H}^{+}\right)$: 905.43 ; Found 905.75 

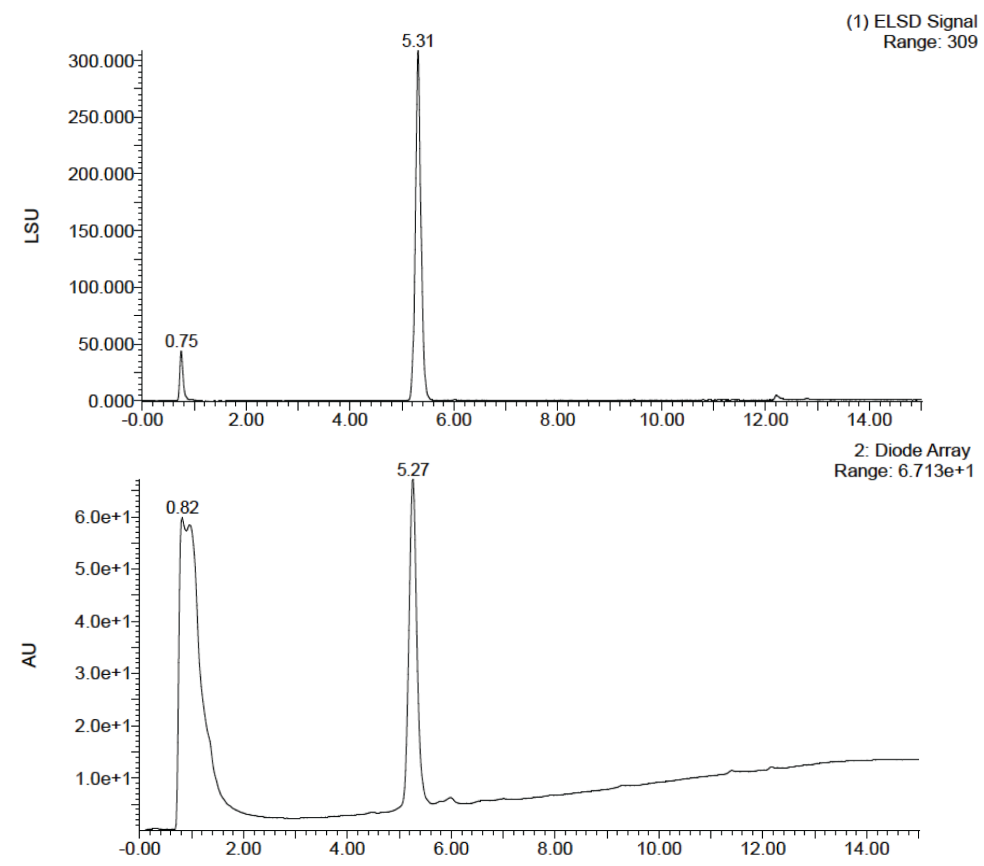

Figure S12. Analytical HPLC trace of MS1 using the general analytical HPLC conditions. The top panel is the signal from the light-scattering detector (ELSD). The bottom panel is absorbance at $254 \mathrm{~nm}$.

\section{Protein Expression and Purification}

\section{A. His-Biotin-NEDD8 Expression and Purification}

HB-NEDD8 was expressed in Escherichia coli and purified following a previously published procedure. ${ }^{5}$

\section{B. Protein Kinase Expression and Purification}

SRC-KD (Chicken, residues 251-533) was a generous gift from the Shokat lab at UCSF. ABL-3D (human, residues 83-534) and SRC-KD(T338I) (Chicken, residues 251-533) were expressed in Escherichia coli and purified following a previously published procedure. ${ }^{6}$ Fulllength SRC (human) was purchased from Thermo Fisher Scientific.

\section{Bcl-xL Expression and Purification}

The gene for Bcl-xL was purchased as a gBlock $^{\circledR}$ from IDT and incorporated into the PMCSG7 vector ${ }^{7}$ using ligation independent cloning. The sequence of the final construct was confirmed by sequencing of the entire gene. The plasmid was transformed into BL21(DE3) $E$. coli cells and a single colony was used to inoculate $1.5 \mathrm{~L}$ of $2 \mathrm{xYT}$ media containing carbenicillin $(100 \mu \mathrm{g} / \mathrm{mL})$. The culture were grown at $37^{\circ} \mathrm{C}$ to an $\mathrm{OD}_{600}$ of $1-1.2$, cooled to $18^{\circ} \mathrm{C}$ for $1 \mathrm{hr}$ and then induced at $18{ }^{\circ} \mathrm{C}$ overnight with $0.5 \mathrm{mM}$ IPTG. Cells were harvested by centrifugation and the pellet were stored at $-80{ }^{\circ} \mathrm{C}$. For protein purification, the pellet was thawed at $0{ }^{\circ} \mathrm{C}$ and then re-suspended in $10 \mathrm{~mL}$ of lysis buffer (50 mM Tris, $\mathrm{pH} 8.0,200 \mathrm{mM} \mathrm{NaCl}, 20 \mathrm{mM}$ imidazole) supplemented with PMSF $(100 \mu \mathrm{g} / \mathrm{mL})$. The cells were lysed using a micro-fludizer and the lysate was cleared by centrifugation at $4{ }^{\circ} \mathrm{C}$. The cleared lysate was added to $400 \mu \mathrm{L}$ of Qiagen Ni-NTA Superflow resin and rotated at $4{ }^{\circ} \mathrm{C}$ for $1 \mathrm{hr}$. The resin was washed $(3 \mathrm{x})$ with lysis buffer and then transferred to a spin column. The purified protein was eluted with elution buffer 
(50 mM Tris, $\mathrm{pH} 8.0,200 \mathrm{mM} \mathrm{NaCl}, 600 \mathrm{mM}$ imidazole). Fractions were analyzed by SDSPAGE and those that were found to be $>95 \%$ pure were pooled, exchanged into storage buffer (25 mM Tris, pH 8.0, $150 \mathrm{mM} \mathrm{NaCl}, 1 \mathrm{mM}$ DTT) and concentrated. The protein was separated into aliquots, snap-frozen and stored at $-80{ }^{\circ} \mathrm{C}$ for later use.

\section{Overlap Extension PCR Method for Generating NEDDylator Fusion Proteins}

General Procedure: SNAP-tag was amplified from the pSNAP-tag (T7)-2 vector (New England Biolabs) using the primers listed. Ubc12 was amplified from previously described plasmids ${ }^{5}$ using the primers listed. The NEDDylator fusions were generated by overlap extension PCR and incorporated into the PMCSG7 vector $^{7}$ using ligation independent cloning. All sequences of the final constructs were confirmed by sequencing of the entire gene.

SNAP-tag primers:

FWD: 5' - TAC TTC CAA TCC AAT GAC AAA GAT TGC GAA ATG AAA -3'

4AA-GlySer linker

RVS: 5'- CTT CAG CGA GAA CAG CTT GAT GCC GCT ACC GCT TCC CAG ACC CGG TTT ACC CAG -3'

10AA-GlySer linker

RVS: 5'- CTT CAG CGA GAA CAG CTT GAT GCC GCT ACC GCT ACC GCT GCC GCT ACC GCT TCC CAG ACC CGG TTT ACC CAG -3'

Ubc12 Primers:

FWD: 5'- GGC ATC AAG CTG TTC TCG CTG AAG -3'

RVS: 5' - TTA TCC ACT TCC AAT GCT ATT TCA GGC AGC GCT CAA AGT A -3'

\section{E. Site-Directed Mutagenesis to Generate NEDDylator(10 $\Delta K)$}

General Procedure: The NEDDylator(S4) gene, located in the pMCSG7, was subjected to sequential mutagenesis using a PCR-based protocol. The vector was amplified using overlapping primers, which contained the desired mutation. After amplification the reactions was incubated with Dpn1 to digest any methylated template DNA. The reaction mixture was then used to transform XL-10 cells. After recovery, the cells were plated and grown in LB agar plates with carbenicillin at $37^{\circ} \mathrm{C}$ overnight. The next day, single colonies from the plates were picked, grown overnight, DNA minipred, and sequenced to check for incorporation of the desired mutation.

The following primers were used for mutagenesis:

K54R

FWD: 5'-CAT GAA ATT CGC CTG CTG GGT AAA GGC ACC AGC-3'

RVS: 5'-ACC CAG CAG GCG AAT TTC ATG CAG GCC CTG TTC G-3'

K153R

FWD: 5'-GCC GCC GTT CGC ACC GCG CTG AGC GGT AAC-3' 
RVS: 5'-CAG CGC GGT GCG AAC GGC GGC GGT GGC-3'

K200Q

FWD: 5'-CGT CTG GGT CAA CCG GGT CTG GGA AGC GG-3'

RVS: 5'-CAG ACC CGG TTG ACC CAG ACG ATG ACC TTC ATG GG-3'

K210R

FWD: 5'-AGC GGC ATC CGC CTG TTC TCG CTG AAG CAG CAG -3'

RVS: 5'-CGA GAA CAG GCG GAT GCC GCT ACC GCT TCC C-3'

K215R

FWD: 5'-TTC TCG CTG CGC CAG CAG AAG AAG GAG GAG GAG TCG-3'

RVS: 5'-CTT CTG CTG GCG CAG CGA GAA CAG GCG GAT GCC-3'

K218R, K219R

FWD: 5'-CGC CAG CAG CGC CGC GAG GAG GAG TCG GCG GGC-3'

RVS: 5'-CTC CTC CTC GCG GCG CTG CTG GCG CAG CGA GAA CAG-3'

K228R

FWD: 5'-GGC GGC ACC CGC GGC AGC AGC CGC CGC GCG-3'

RVS: 5'-GCT GCT GCC GCG GGT GCC GCC CGC CGA CTC-3'

K232R, K233R

FWD: 5'-GGC AGC AGC CGC CGC GCG TCG GCG GCG CAG CTG-3'

RVS: 5'-CGC CGA CGC GCG GCG GCT GCT GCC CTT GGT GCC-3'

\section{F. PCR Method for Generating NEDDylator(7AK)-BAD}

General Procedure: Ubc12 $(7 \Delta K)$ was amplified from the NEDDylator(10 $\Delta K)$ plasmid using the primers listed. The NEDDylator( $7 \Delta \mathrm{K})$-BAD gene was generated in three sequential PCR reactions and incorporated into the PMCSG7 vector ${ }^{7}$ using ligation independent cloning. The sequence of the final construct was confirmed by sequencing of the entire gene.

Ubc12(7 $\Delta \mathrm{K})$ Primers:

RVS: 5'- GAA TTC GGA TCC GTT ATC CAC TTC CAA TCT ATT TCA GGC AGC GCT CAA AGT AGG -3'

Reaction 1 FWD:

5'-CCG ATG AAT TCG AAG GCT CGT TCG CAG CGA GCG GTA GCG GCA TCC GCC TGT TCT CGC TGC-3'

Reaction 2 FWD:

5'-GCG GCC CAA CGC TAT GGG CGC GAA CTG CGC CGT ATG TCC GAT GAA TTC GAA GGC TCG TTC-3' 
Reaction 3 FWD:

5'- ACC GAG AAC CTG TAC TTC CAA TCC AAT CTC TGG GCG GCC CAA CGC TAT GGG-3'

\section{G. NEDDylator Expression and Purification}

Plasmids were transformed into BL21(DE3) E. coli cells and a single colony was used to inoculate $1.5 \mathrm{~L}$ of $2 \times Y T$ media containing carbenicillin $(100 \mu \mathrm{g} / \mathrm{mL})$. Cultures were grown at $37{ }^{\circ} \mathrm{C}$ to an $\mathrm{OD}_{600}$ of $1-1.2$, cooled to $18^{\circ} \mathrm{C}$ for $1 \mathrm{hr}$ and then induced at $18{ }^{\circ} \mathrm{C}$ overnight with 0.5 $\mathrm{mM}$ IPTG. Cells were harvested by centrifugation and the pellets were stored at $-80{ }^{\circ} \mathrm{C}$. For protein purification, the pellets were thawed at $0{ }^{\circ} \mathrm{C}$ and then re-suspended in $10 \mathrm{~mL}$ of NEDDylator lysis buffer (50 mM Tris, $\mathrm{pH} 8.0,200 \mathrm{mM} \mathrm{NaCl}, 20 \mathrm{mM}$ imidazole) supplemented with PMSF $(100 \mu \mathrm{g} / \mathrm{mL})$. The cells were lysed using a micro-fludizer and the lysate was cleared by centrifugation at $4{ }^{\circ} \mathrm{C}$. The cleared lysate was added to $400 \mu \mathrm{L}$ of Qiagen Ni-NTA Superflow resin and rotated at $4{ }^{\circ} \mathrm{C}$ for $1 \mathrm{hr}$. The resin was washed $(3 \mathrm{x})$ with lysis buffer and then transferred to a spin column. The purified protein was eluted with NEDDylator elution buffer (50 $\mathrm{mM}$ Tris, $\mathrm{pH}$ 8.0, $200 \mathrm{mM} \mathrm{NaCl}, 600 \mathrm{mM}$ imidazole). Fractions were analyzed by SDS-PAGE and those that were found to be $>95 \%$ pure were pooled, exchanged into storage buffer $(25 \mathrm{mM}$ Tris, $\mathrm{pH} 8.0,150 \mathrm{mM} \mathrm{NaCl}, 1 \mathrm{mM}$ DTT) and concentrated. The protein was separated into aliquots, snap-frozen and stored at $-80{ }^{\circ} \mathrm{C}$ for later use. All proteins were analyzed with either a Water LCT Premier or Waters Xevo G2-XS Mass Spectrometer.

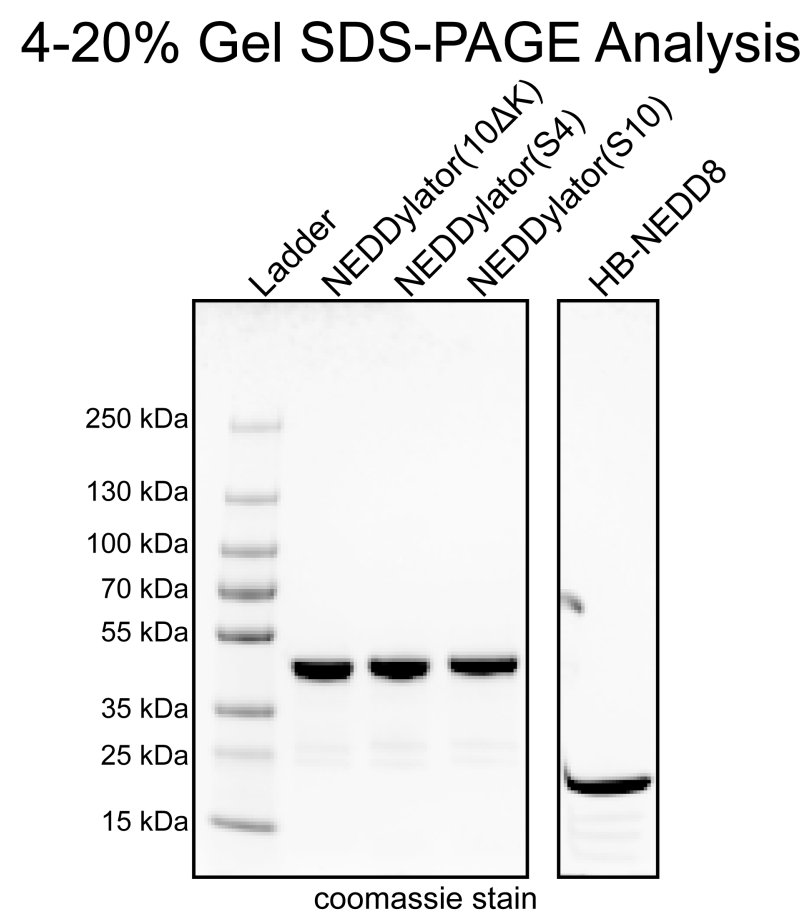

Figure S13. SDS-PAGE analysis of NEDDylator fusions and HB-NEDD8. $3 \mu \mathrm{g}$ of each NEDDylator fusion protein and HB-NEDD8 was loaded onto a 4-20\% gradient gel in 1X Laemmli loading buffer containing 2-mercaptoethanol. The gel was run at $200 \mathrm{~V}$ for $33 \mathrm{~min}$. The gel was stained with coomassie for $30 \mathrm{sec}$ in the microwave and then an additional 5 min while shaking. The gel was then destained for 3 hours. 
Mass Spectrometry Analysis of Expressed NEDDylator Proteins

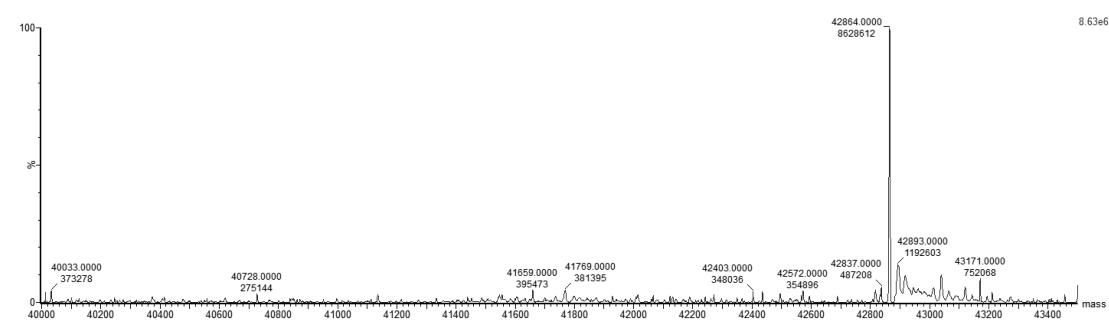

Figure S14. Mass spectrum of NEDDylator(S4). Calculated for $\mathrm{C}_{1923} \mathrm{H}_{3005} \mathrm{~N}_{521} \mathrm{O}_{564} \mathrm{~S}_{13}$ : 42863.9, Observed: 42864.0

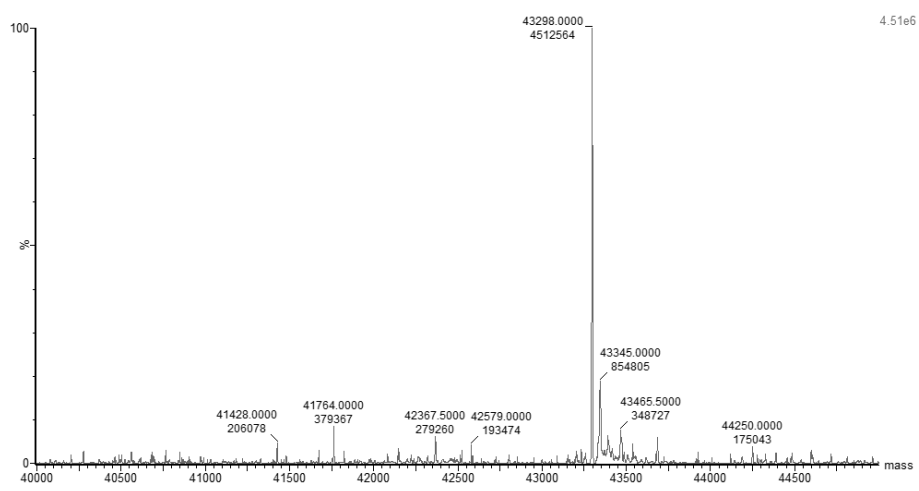

Figure S15. Mass spectrum of NEDDylator(S10). Calculated for $\mathrm{C}_{1938} \mathrm{H}_{3029} \mathrm{~N}_{527} \mathrm{O}_{573} \mathrm{~S}_{13}$ : 43296.3, Observed: 43298.0

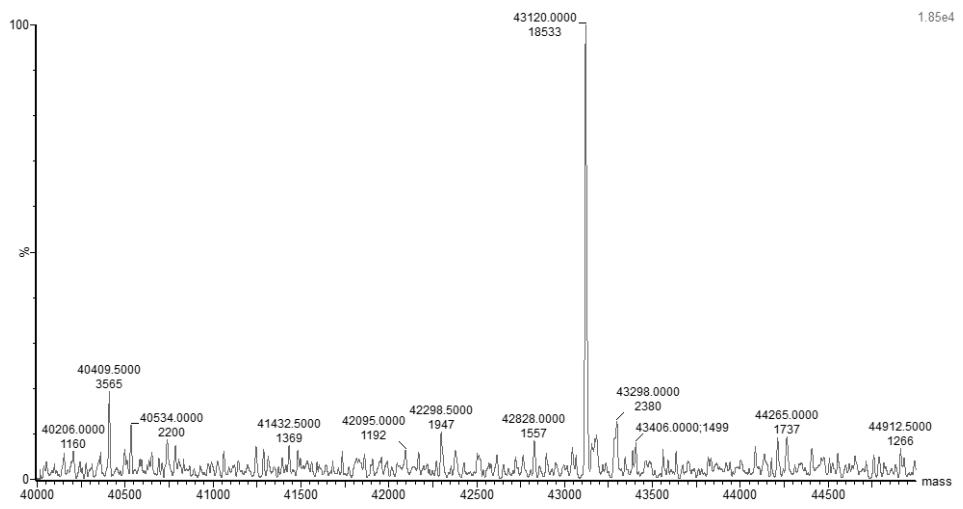

Figure S16. Mass spectrum of NEDDylator $(10 \Delta \mathrm{K})$. Calculated for $\mathrm{C}_{1922} \mathrm{H}_{3001} \mathrm{~N}_{539} \mathrm{O}_{565} \mathrm{~S}_{13}$ : 43116, Observed: 43120.0 


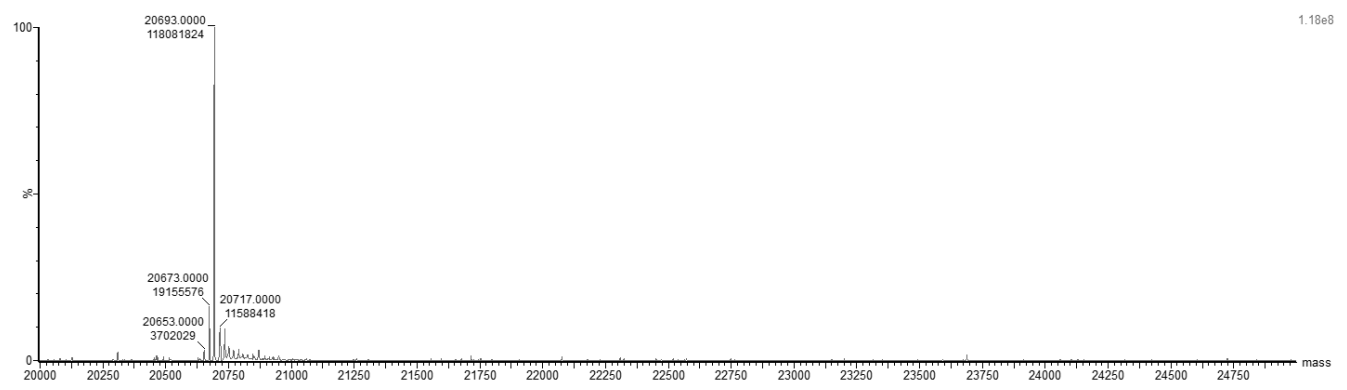

Figure S17. Mass spectrum of HB-NEDD8. Calculated for $\mathrm{C}_{907} \mathrm{H}_{1495} \mathrm{~N}_{254} \mathrm{O}_{284} \mathrm{~S}_{6}:$ 20693.6, Observed: 20693.0

\section{H. Preparation and Purification of NEDDylator-Small Molecule Chimeras}

\section{Preparation of NEDDylator(S4)-DS1, NEDDylator(S10)-DS1, NEDDylator(10 $\Delta K)$-DS1,} and NEDDylator(10 $\Delta K)-M S 1$

NEDDylator(S4), NEDDylator(S10), and NEDDylator(10 $\Delta \mathrm{K})$ were labeled with DS1, using the following conditions: NEDDylator(S4), NEDDylator(S10), or NEDDylator(10 $\Delta K)(15$ $\mu \mathrm{M})$ was incubated with DS1 $(60 \mu \mathrm{M})$ or MS1 $(25 \mu \mathrm{M})$ in labeling buffer $(50 \mathrm{mM}$ Tris buffer, $\mathrm{pH} 7.5,100 \mathrm{nM} \mathrm{NaCl}$ and $1 \mathrm{mM}$ DTT) for $2 \mathrm{~h}$ at room temperature. The protein-small molecule conjugate was then purified (using the conditions described below), quantified, snap-frozen in $\mathrm{LN}_{2}$ and stored at $-80{ }^{\circ} \mathrm{C}$ until used in activity or NEDDylation assays.

\section{Purification of NEDDylator(S4)-DS1, NEDDylator(S10)-DS1, and NEDDylator(10 $\Delta K)-D S 1$ Conjugates}

For NEDDylator(S4)-DS1, NEDDylator(S10)-DS1， NEDDylator(10 K)-DS1, and NEDDylator(10 $10 \mathrm{~K})$-MS1 to function correctly it is necessary to remove any unreacted DS1 or MS1. All small-scale labeling reactions were purified 2 times using, $0.5 \mathrm{~mL}, 7 \mathrm{~K}$ MWCO, Zeba $^{\mathrm{TM}}$ Spin Desalting Columns according to the manufacture's protocol. Large-scale labeling reactions were purified using a Superdex 200 10/300 GL column (GE). The concentration of the eluted protein in both cases was determined using a Thermo Scientific Nanodrop to measure absorbance at $280 \mathrm{nM}$. Percent labeling was analyzed with either a Water LCT Premier or Waters Xevo G2-XS Mass Spectrometer and found to be $>90 \%$ for all proteins. Purified AGTsmall molecule conjugates were either used directly in activity and NEDDylation assays or snapfrozen in $\mathrm{LN}_{2}$ and stored at $-80{ }^{\circ} \mathrm{C}$ until used. 
Mass Spectrometry Analysis of NEDDylator(S4)-DS1, NEDDylator(S10)-DS1, and NEDDylator(10 $\Delta K)$-DS1

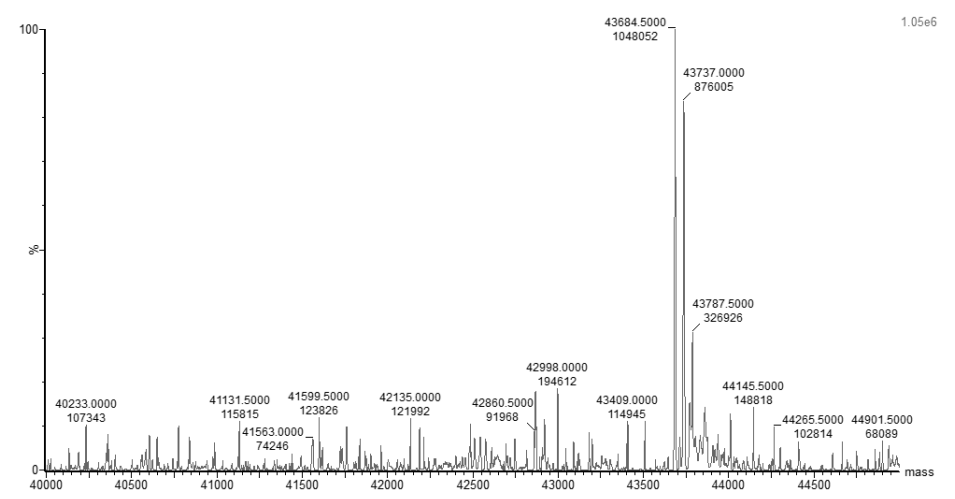

Figure S18. Mass spectrum of NEDDylator(S4)-DS1: Calculated for $\mathrm{C}_{1963} \mathrm{H}_{3055} \mathrm{ClN}_{531} \mathrm{O}_{569} \mathrm{~S}_{14}$ : 43681.2, Observed: 43684.5

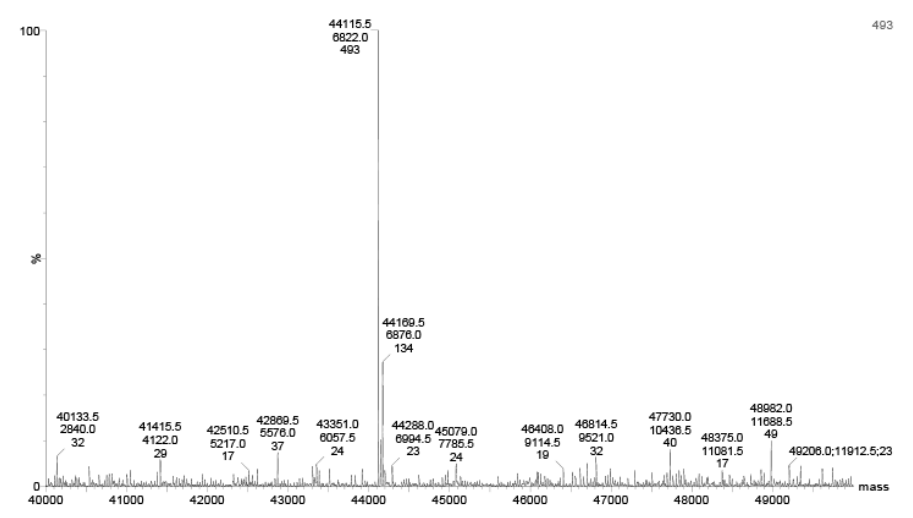

Figure S19. Mass spectrum of NEDDylator(S10)-DS1: Calculated for $\mathrm{C}_{1978} \mathrm{H}_{3079} \mathrm{ClN}_{537} \mathrm{O}_{578} \mathrm{~S}_{14}$ : 44113.6, Observed: 44115.5

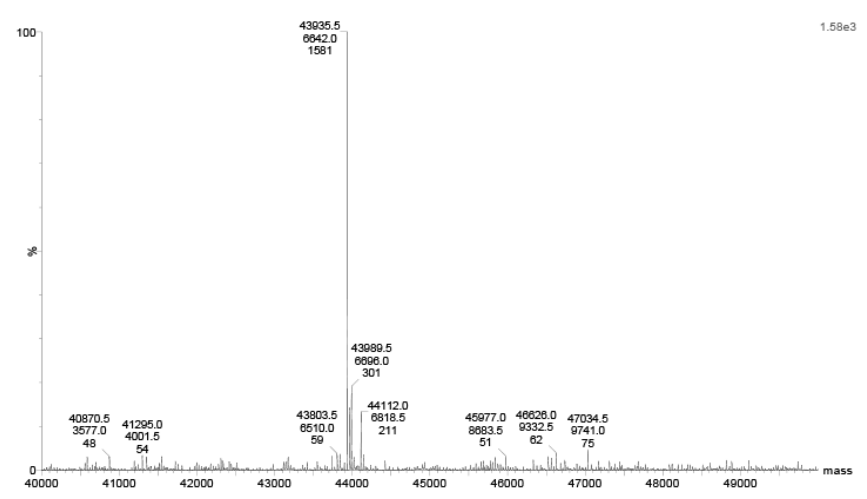

Figure S20.Mass spectrum of NEDDylator(10 $\mathrm{K})$-DS1: Calculated for $\mathrm{C}_{1962} \mathrm{H}_{3051} \mathrm{ClN}_{549} \mathrm{O}_{570} \mathrm{~S}_{14}$ : 43933.3, Observed: 43935.5 


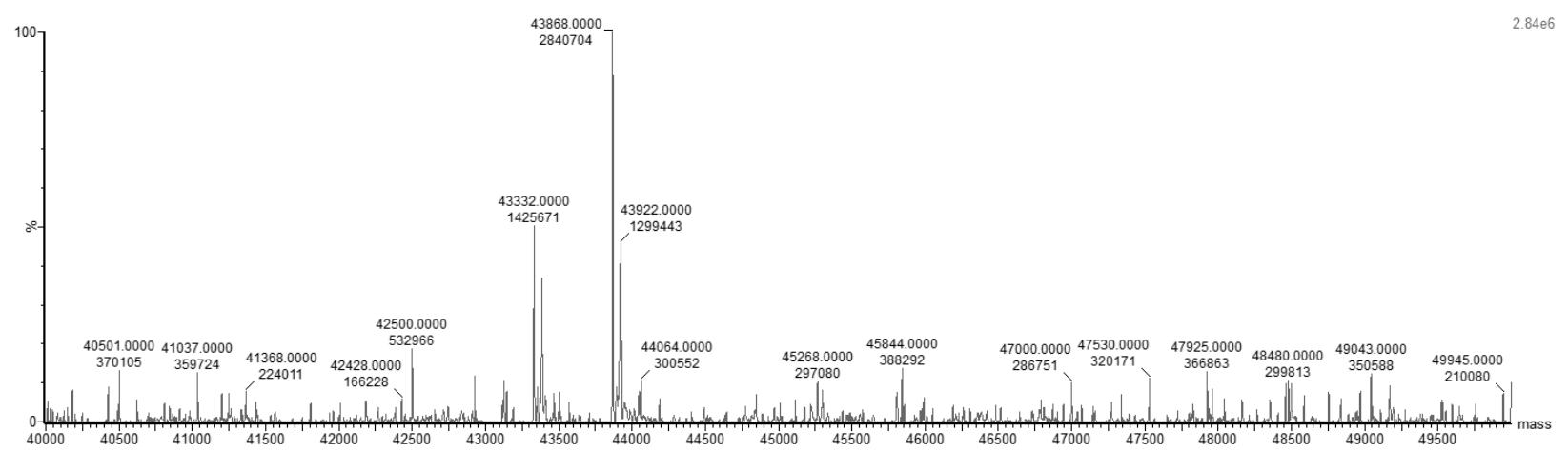

Figure S21.Mass spectrum of NEDDylator(10 KK)-MS1: Calculated for $\mathrm{C}_{1960} \mathrm{H}_{3049} \mathrm{~N}_{550} \mathrm{O}_{571} \mathrm{~S}_{13}$ : 43870.25, Observed: 43868.0

\section{In Vitro Kinase Activity Assays}

Activity Assay conditions for dasatinib and DS1 against ABL-3D

Dasatinib and DS1 (initial concentration $=30 \mu \mathrm{M}$ of dasatinib and DS1, 3-fold serial dilutions, 10 data points) was assayed in quadruplicate against ABL-3D (final concentration $=10$ $\mathrm{nM}$ ) in assay buffer containing $75 \mathrm{mM}$ HEPES, $\mathrm{pH}=7.5,15 \mathrm{mM} \mathrm{MgCl} 2,3.75 \mathrm{mM}$ EGTA, 150 $\mathrm{mM} \mathrm{NaCl}, 0.2 \mathrm{mg} / \mathrm{mL}$ BSA, $\gamma^{32} \mathrm{P}$ ATP $(0.2 \mu \mathrm{Ci} /$ well $)$ and an optimized ABL peptide substrate of the sequence Ac-EAIYAAPFAKKK-OH (final concentration $=50 \mu \mathrm{M}$ ) The final volume of each assay well was $30 \mu \mathrm{L}$. The enzymatic reaction was run at room temperature for 1 hour and then terminated by spotting $4.6 \mu \mathrm{L}$ of the reaction mixture onto a phosphocellulose membrane. Membranes were washed with $0.5 \%$ phosphoric acid ( $4 \mathrm{x}, 10$ minutes each wash), dried, and the radioactivity was determined by phosphorimaging with an Amersham Biosciences Typhoon Trio phosphor scanner. The scanned membranes were quantified with ImageQuant and converted to percent inhibition. Data was analyzed using Prism Graphpad software and $\mathrm{IC}_{50}$ values were determined using non-linear regression analysis.
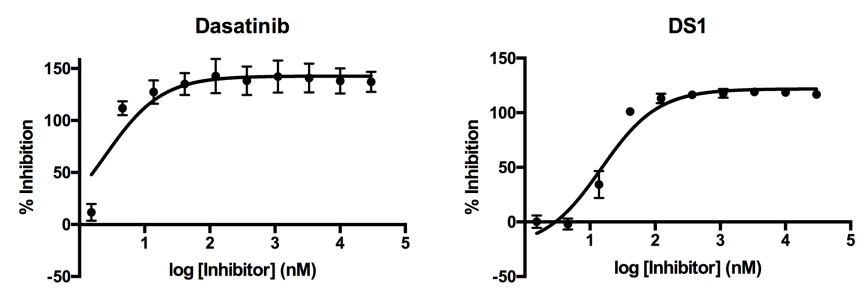

Figure S22. Representative in vitro kinase assay data for dasatinib and DS1 against ABL-3D.

\section{Activity Assay conditions for NEDDylator(S4)-DS1 and NEDDylator(S10)-DS1 against} ABL-3D

NEDDylator(S4)-DS1 and NEDDylator(S10)-DS1 (initial concentration $=3-6 \mu \mathrm{M}, 3$-fold serial dilutions, 10 data points) were assayed in quadruplicate against ABL-3D (final concentration $=10 \mathrm{nM}$ ) in assay buffer containing $75 \mathrm{mM}$ HEPES, $\mathrm{pH}=7.5,15 \mathrm{mM} \mathrm{MgCl}$, $3.75 \mathrm{mM}$ EGTA, $150 \mathrm{mM} \mathrm{NaCl}, 0.2 \mathrm{mg} / \mathrm{mL}$ BSA, $\gamma^{32} \mathrm{P}$ ATP $(0.2 \mu \mathrm{Ci} /$ well $)$ and an optimized ABL peptide substrate of the sequence Ac-EAIYAAPFAKKK-OH (final concentration $=50$ $\mu \mathrm{M})$ The final volume of each assay well was $30 \mu \mathrm{L}$. The enzymatic reaction was run at room temperature for 1 hour and then terminated by spotting $4.6 \mu \mathrm{L}$ of the reaction mixture onto a 
phosphocellulose membrane. Membranes were washed with $0.5 \%$ phosphoric acid $(4 \mathrm{x}, 10$ minutes each wash), dried, and the radioactivity was determined by phosphorimaging with an Amersham Biosciences Typhoon Trio phosphor scanner. The scanned membranes were quantified with ImageQuant and converted to percent inhibition. Data was analyzed using Prism Graphpad software and $\mathrm{IC}_{50}$ values were determined using non-linear regression analysis.
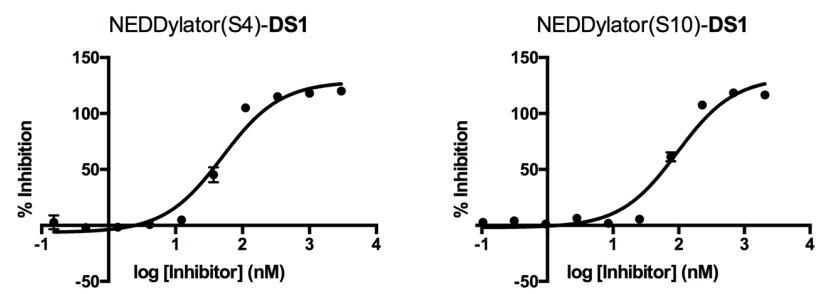

Figure S23. Representative in vitro kinase assay data for NEDDylator(S4)-DS1 and NEDDylator(S10)-DS1 against ABL-3D.

\section{Activity Assay conditions for dasatinib and DS1 against SRC-KD}

Dasatinib and DS1 (initial concentration $=30 \mu \mathrm{M}$, 3-fold serial dilutions, 10 data points) were assayed in quadruplicate against SRC-KD (final concentration $=1 \mathrm{nM}$ ) in assay buffer containing $75 \mathrm{mM}$ HEPES, $\mathrm{pH}=7.5,15 \mathrm{mM} \mathrm{MgCl}_{2}, 3.75 \mathrm{mM}$ EGTA, $150 \mathrm{mM} \mathrm{NaCl}, 0.2$ $\mathrm{mg} / \mathrm{mL}$ BSA, $\gamma^{32} \mathrm{P}$ ATP $(0.2 \mu \mathrm{Ci} /$ well $)$ and an optimized SRC peptide substrate of the sequence Ac-EIYGEFKKK-OH (final concentration $=50 \mu \mathrm{M}$ ). The final volume of each assay well was $30 \mu \mathrm{L}$. The enzymatic reaction was run at room temperature for 30 minutes and then terminated by spotting $4.6 \mu \mathrm{L}$ of the reaction mixture onto a phosphocellulose membrane. Membranes were washed with $0.5 \%$ phosphoric acid (4x, 10 minutes each wash), dried, and the radioactivity was determined by phosphorimaging with an Amersham Biosciences Typhoon Trio phosphor scanner. The scanned membranes were quantified with ImageQuant and converted to percent inhibition. Data was analyzed using Prism Graphpad software and $\mathrm{IC}_{50}$ values were determined using non-linear regression analysis.
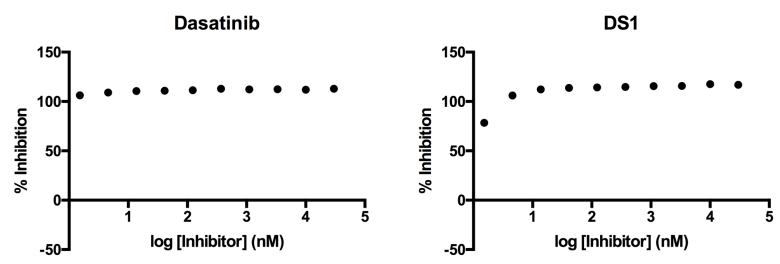

Figure S24. Representative in vitro kinase assay data for dasatinib and DS1 against SRC-KD.

\section{Activity Assay Conditions for NEDDylator(S4)-DS1 and NEDDylator(S10)-DS1 against SRC-KD}

NEDDylator(S4)-DS1 and NEDDylator(S10)-DS1 (initial concentration $=3 \mu \mathrm{M}, 3$-fold serial dilutions, 10 data points) was assayed in quadruplicate against SRC-KD (final concentration $=1 \mathrm{nM}$ ) in assay buffer containing $75 \mathrm{mM}$ HEPES, $\mathrm{pH}=7.5,15 \mathrm{mM} \mathrm{MgCl} 2,3.75$ $\mathrm{mM}$ EGTA, $150 \mathrm{mM} \mathrm{NaCl}, 0.2 \mathrm{mg} / \mathrm{mL}$ BSA, $\gamma^{32} \mathrm{P}$ ATP $(0.2 \mu \mathrm{Ci} /$ well $)$ and an optimized SRC peptide substrate of the sequence Ac-EIYGEFKKK-OH (final concentration $=100 \mu \mathrm{M}$ ). The final volume of each assay well was $30 \mu \mathrm{L}$. The enzymatic reaction was run at room temperature for 30 minutes and then terminated by spotting $4.6 \mu \mathrm{L}$ of the reaction mixture onto a phosphocellulose membrane. Membranes were washed with $0.5 \%$ phosphoric acid $(4 \mathrm{x}, 10$ 
minutes each wash), dried, and the radioactivity was determined by phosphorimaging with an Amersham Biosciences Typhoon Trio phosphor scanner. The scanned membranes were quantified with ImageQuant and converted to percent inhibition. Data was analyzed using Prism Graphpad software and $\mathrm{IC}_{50}$ values were determined using non-linear regression analysis.
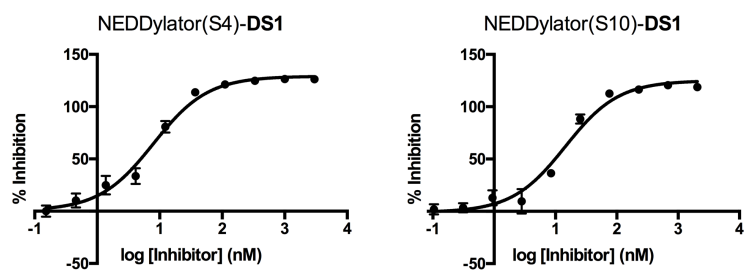

Figure S25. Representative in vitro kinase assay data for NEDDylator(S4)-DS1 and NEDDylator(S10)-DS1 against SRC-KD.

\section{Activity Assay Conditions for DS1 against SRC-KD(T338I)}

Inhibitor DS1 (initial concentration $=30 \mu \mathrm{M}$, 3-fold serial dilutions, 10 data points) was assayed in quadruplicate against SRC-KD(T338I) (final concentration $=1 \mathrm{nM}$ ) in assay buffer containing $75 \mathrm{mM}$ HEPES, $\mathrm{pH}=7.5,15 \mathrm{mM} \mathrm{MgCl}_{2}, 3.75 \mathrm{mM}$ EGTA, $150 \mathrm{mM} \mathrm{NaCl}, 0.2$ $\mathrm{mg} / \mathrm{mL}$ BSA, $\gamma^{32} \mathrm{P}$ ATP $(0.2 \mu \mathrm{Ci} /$ well $)$ and an optimized SRC peptide substrate of the sequence Ac-EIYGEFKKK-OH (final concentration $=100 \mu \mathrm{M}$ ). The final volume of each assay well was $30 \mu \mathrm{L}$. The enzymatic reaction was run at room temperature for 30 minutes and then terminated by spotting $4.6 \mu \mathrm{L}$ of the reaction mixture onto a phosphocellulose membrane. Membranes were washed with $0.5 \%$ phosphoric acid ( $4 \mathrm{x}, 10$ minutes each wash), dried, and the radioactivity was determined by phosphorimaging with an Amersham Biosciences Typhoon Trio phosphor scanner. The scanned membranes were quantified with ImageQuant and converted to percent inhibition. Data was analyzed using Prism Graphpad software and $\mathrm{IC}_{50}$ values were determined using non-linear regression analysis.

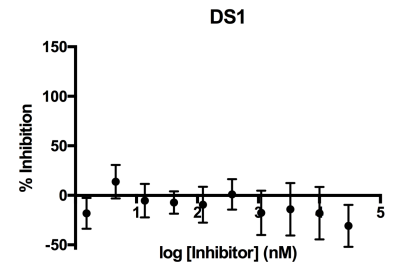

Figure S26. Representative in vitro kinase assay data for DS1 against SRC-KD(T338I).

\section{NEDDylation Assay}

\section{A. Assay Conditions for NEDDylation of Recombinant Protein}

\section{NEDDylator Titration Assays}

NEDDylator(S4)-DS1 and NEDDylator(S10)-DS1 (initial concentration $=2.5 \mu \mathrm{M}, 2$-fold serial dilutions, 10 data points) were incubated with ABL-3D (200 nM final concentration) in NEDDylation assay buffer containing $50 \mathrm{mM}$ Tris $\mathrm{pH}$ 8.0, $10 \mu \mathrm{M}$ HB-NEDD8, $50 \mathrm{nM}$ NAE1/UBA3, $1 \mathrm{mg} / \mathrm{ml} \mathrm{BSA,} 5 \mathrm{mM}$ ATP, and $10 \mathrm{mM} \mathrm{MgCl}$. The final volume of each assay well was $10 \mu \mathrm{L}$. The enzymatic reaction was run at $37^{\circ} \mathrm{C}$ for 2 hours and then terminated by addition of $3 \mu \mathrm{L}$ of $4 \mathrm{X}$ Laemmli loading buffer containing 2-mercaptoethanol followed by 
boiling for 5 minutes. The samples were placed at $-20{ }^{\circ} \mathrm{C}$ until being subjected to SDS-PAGE, transferred to nitrocellulose and probed for ABL ( $\alpha-A B L, A 5844$, Sigma Aldrich). Successful NEDDylation was observed as appearance of a NEDDylation ladder when probing for ABL.

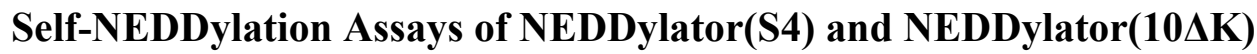

NEDDylator(S4) and NEDDylator $(10 \Delta \mathrm{K})($ final concentration $=1 \mu \mathrm{M})$ were incubated in NEDDylation assay buffer containing $50 \mathrm{mM}$ Tris $\mathrm{pH}$ 8.0, $10 \mu \mathrm{M}$ HB-NEDD8, $50 \mathrm{nM}$ NAE1/UBA3, $1 \mathrm{mg} / \mathrm{ml} \mathrm{BSA,} 5 \mathrm{mM}$ ATP, and $10 \mathrm{mM} \mathrm{MgCl}_{2}$. The final volume of each assay well was $20 \mu \mathrm{L}$. The enzymatic reaction was run at $37{ }^{\circ} \mathrm{C}$ for 2 hours and then terminated by addition of $7 \mu \mathrm{L}$ of $4 \mathrm{X}$ Laemmli loading buffer containing 2-mercaptoethanol followed by boiling for 5 minutes. The samples were placed at $-20{ }^{\circ} \mathrm{C}$ until being subjected to SDS-PAGE, transferred to nitrocellulose and probed UBC-12 ( $\alpha$-Ubc12, sc-100608, Santa Cruz Biotechnology). The intensity of all bands, both of the NEDDylated and unmodified NEDDylator was quantified using ImageJ. The percent of self-NEDDylation was calculated by dividing the summed intensity of the higher molecular weight by the summed intensity of all bands. The assay was performed in triplicate.

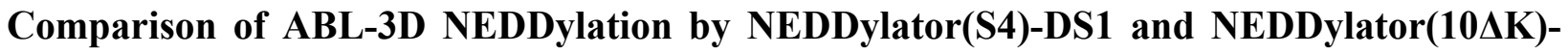 DS1}

NEDDylator(S4)-DS1 and NEDDylator(10 $1 \mathrm{~K}$ )-DS1 (final concentration $=1 \mu \mathrm{M}$ ) were incubated with ABL-3D (200 nM final concentration) in NEDDylation assay buffer containing $50 \mathrm{mM}$ Tris $\mathrm{pH}$ 8.0, $10 \mu \mathrm{M}$ HB-NEDD8, $50 \mathrm{nM}$ NAE1/UBA3, $1 \mathrm{mg} / \mathrm{ml} \mathrm{BSA,} 5 \mathrm{mM}$ ATP, and $10 \mathrm{mM} \mathrm{MgCl}_{2}$. The final volume of each assay well was $20 \mu \mathrm{L}$. The enzymatic reaction was run at $37{ }^{\circ} \mathrm{C}$ for 2 hours and then terminated by addition of $7 \mu \mathrm{L}$ of $4 \mathrm{X}$ Laemmli loading buffer containing 2-mercaptoethanol followed by boiling for 5 minutes. The samples were placed at $20{ }^{\circ} \mathrm{C}$ until being subjected to SDS-PAGE, transferred to nitrocellulose and probed ABL $(\alpha-$ ABL, A5844, Sigma Aldrich). The intensity of all bands, both of the NEDDylated and unmodified ABL-3D was quantified using ImageJ. The percent of ABL-3D NEDDylation was calculated by dividing the summed intensity of the higher molecular weight by the summed intensity of all bands. The assay was performed in triplicate.

\section{NEDDylation Dasatinib Competition Assays}

NEDDylator $(10 \Delta \mathrm{K})$-DS1 (final concentration $=1 \mu \mathrm{M})$ was incubated with ABL-3D (200 $\mathrm{nM}$ final concentration), SRC-KD (200 nM final concentration), SRC-KD(T338I) (200 nM final concentration), or full-length SRC (200 nM final concentration) in NEDDylation assay buffer containing $50 \mathrm{mM}$ Tris $\mathrm{pH}$ 8.0, $10 \mu \mathrm{M}$ HB-NEDD8, $1 \mathrm{mg} / \mathrm{ml} \mathrm{BSA}, 5 \mathrm{mM}$ ATP, $10 \mathrm{mM} \mathrm{MgCl}$, $0.1 \mathrm{mM}$ DTT, and 2.5\% DMSO in the presence or absence of $50 \mathrm{nM} \mathrm{NAE1/UBA3}$ and the presence or absence of $10 \mu \mathrm{M}$ dasatinib. The final volume of each assay well was $20 \mu \mathrm{L}$. The enzymatic reaction was run at $37^{\circ} \mathrm{C}$ for 2 hours and then terminated by addition of $7 \mu \mathrm{L}$ of $4 \mathrm{X}$ Laemmli loading buffer containing 2-mercaptoethanol followed by boiling for 5 minutes. The samples were placed at $-20^{\circ} \mathrm{C}$ until being subjected to SDS-PAGE, transferred to nitrocellulose and probed for either ABL-3D ( $\alpha$-ABL, A5844, Sigma Aldrich), SRC-KD ( $\alpha$-SRC, sc-18, Santa Cruz Biotechnology), or full-length SRC ( $\alpha$-SRC, L4A1, Cell Signaling Technology). Successful 
NEDDylation was observed as appearance of a NEDDylation ladder when probing for ABL or SRC.

\section{DHFR NEDDylation Assay}

NEDDylator $(10 \Delta \mathrm{K}$ )-MS1 (final concentration $=1 \mu \mathrm{M}$ ) was incubated with DHFR (200 nM final concentration) in NEDDylation assay buffer containing $50 \mathrm{mM}$ Tris $\mathrm{pH}$ 8.0, $10 \mu \mathrm{M}$ HB-NEDD8, $1 \mathrm{mg} / \mathrm{ml} \mathrm{BSA,} 5 \mathrm{mM}$ ATP, $10 \mathrm{mM} \mathrm{MgCl}_{2}, 0.1 \mathrm{mM}$ DTT, and 2.5\% DMSO in the presence or absence of $50 \mathrm{nM}$ NAE1/UBA3 and the presence or absence of $10 \mu \mathrm{M}$ methotrexate. The final volume of each assay well was $20 \mu \mathrm{L}$. The enzymatic reaction was run at $37^{\circ} \mathrm{C}$ for 2 hours and then terminated by addition of $7 \mu \mathrm{L}$ of $4 \mathrm{X}$ Laemmli loading buffer containing 2-mercaptoethanol followed by boiling for 5 minutes. The samples were placed at $-20{ }^{\circ} \mathrm{C}$ until being subjected to SDS-PAGE, transferred to nitrocellulose and probed for DHFR ( $\alpha$-DHFR, EPR5285, Abcam). Successful NEDDylation was observed as appearance of a NEDDylation ladder when probing for DHFR.

\section{Bcl-xL NEDDylation Assay}

NEDDylator(7 $\triangle \mathrm{K}$ )-BAD (final concentration $=200 \mathrm{nM}$ ) was incubated with Bcl-xL (100 nM final concentration) in NEDDylation assay buffer containing $50 \mathrm{mM}$ Tris $\mathrm{pH} 8.0,10 \mu \mathrm{M} \mathrm{HB}$ NEDD8, $1 \mathrm{mg} / \mathrm{ml}$ BSA, $5 \mathrm{mM}$ ATP, $10 \mathrm{mM} \mathrm{MgCl} 2,0.1 \mathrm{mM}$ DTT, and $2.5 \%$ DMSO in the presence or absence of $50 \mathrm{nM}$ NAE1/UBA3 and the presence or absence of $30 \mu \mathrm{M}$ ABT-737 or ABT-263. The final volume of each assay well was $20 \mu \mathrm{L}$. The enzymatic reaction was run at $37{ }^{\circ} \mathrm{C}$ for 2 hours and then terminated by addition of $7 \mu \mathrm{L}$ of 4 X Laemmli loading buffer containing 2-mercaptoethanol followed by boiling for 5 minutes. The samples were placed at $20{ }^{\circ} \mathrm{C}$ until being subjected to SDS-PAGE, transferred to nitrocellulose and probed for Bcl-xL ( $\alpha$-Bcl-xL, 54H6, Cell Signaling Technology). Successful NEDDylation was observed as appearance of a NEDDylation ladder when probing for Bcl-xL.

\section{B. Assay Conditions for NEDDylation of Cell Lysates}

A549 cells were acquired from the Cell Culture Facility at UCSF and cultured in Gibco ${ }^{\mathrm{TM}}$ F$12 \mathrm{~K}$ Kaighn's Modification media supplemented with $10 \%$ fetal bovine serum and penicillin/streptomycin. Cells were incubated at $37{ }^{\circ} \mathrm{C}$ and $5 \% \mathrm{CO}_{2}$. A549 cells were lysed by incubation with cold lysis buffer $(50 \mathrm{mM}$ Tris- $\mathrm{HCl} \mathrm{pH} 7.5,100 \mathrm{mM} \mathrm{NaCl}, 5 \%$ glycerol, $1.5 \mathrm{mM}$ $\mathrm{MgCl}_{2}, 25 \mathrm{mM} \mathrm{NaF}, 1 \mathrm{mM} \mathrm{Na} \mathrm{VO}_{4}, 1 x$ protease inhibitor cocktail (Cell Signaling Technology), 1x phosphatase inhibitor cocktail, $1 \mathrm{mM}$ PMSF) on ice for 20 minutes. The lysate was then cleared by centrifugation at $14000 \mathrm{rpm}$ for 1 hour at $4{ }^{\circ} \mathrm{C}$. The lysate concentration was quantified by BCA assay.

NEDDylator $(10 \Delta \mathrm{K}$ )-DS1 (final concentration $=1 \mu \mathrm{M}$ ) was incubated with A549 lysate (5 $\mathrm{mg} / \mathrm{mL}$ final concentration) in NEDDylation assay buffer containing $50 \mathrm{mM}$ Tris $\mathrm{pH} 8.0,10 \mu \mathrm{M}$ HB-NEDD8, $50 \mathrm{nM}$ NAE1/UBA3, $5 \mathrm{mM}$ ATP, $10 \mathrm{mM} \mathrm{MgCl}_{2}, 0.1 \mathrm{mM}$ DTT, and 2.5\% DMSO in the presence or absence of $10 \mu \mathrm{M}$ dasatinib. The final volume of each assay well was $50 \mu \mathrm{L}$. The enzymatic reaction was run at $37{ }^{\circ} \mathrm{C}$ for 18 hours and then terminated by addition of $16 \mu \mathrm{L}$ of 4X Laemmli loading buffer containing 2-mercaptoethanol followed by boiling for 5 minutes. 
The samples were placed at $-20{ }^{\circ} \mathrm{C}$ until being subjected to SDS-PAGE, transferred to nitrocellulose and probed for SRC ( $\alpha$-SRC, L4A1, Cell Signaling Technology). Successful NEDDylation was observed as appearance of a NEDDylation ladder when probing for SRC.

\section{NEDDylation and Affinity Capture of NEDDylated Proteins From SILAC A549 Cell Lysates}

$65 \mathrm{mg}$ of L-Lys and $42 \mathrm{mg}$ of $\mathrm{L}-\mathrm{Arg} \bullet \mathrm{HCl}$ were added to 0.5 liter of -Lys/-Arg SILAC DMEM (Thermo Fisher) supplemented with $10 \%$ dialyzed fetal bovine serum (FBS) (Thermo Fisher) and penicillin/streptomycin to generate "Light" medium. $90.6 \mathrm{mg}$ of ${ }^{13} \mathrm{C}_{6}{ }^{15} \mathrm{~N}_{2} \mathrm{~L}$ Lys $\bullet 2 \mathrm{HCl}$ and $43.9 \mathrm{mg}$ of ${ }^{13} \mathrm{C}_{6}{ }^{15} \mathrm{~N}_{4} \mathrm{~L}-\mathrm{Arg} \bullet \mathrm{HCl}$ (Cambridge Isotope) were added to the same amount and composition of medium to generate "Heavy" medium. A549 cells were acquired from the Cell Culture Facility at UCSF and cultured separately in "Light" or "Heavy" medium for 3 passages ( $\sim 9$ days) to allow for thorough isotope incorporation. Cells were incubated at $37{ }^{\circ} \mathrm{C}$ and $5 \% \mathrm{CO}_{2}$. "Heavy" and "Light" $\mathrm{A} 549$ cells were lysed separately as described above to generate "Heavy" and "Light" lysate. After clearing, both lysates were desalted using PD-10 columns (GE) while following the manufacture's recommended protocol.

Separate "Heavy" and "Light" NEDDylation reactions utilizing NEDDylator(10 $\Delta$ K)-DS1 were performed as described above (2.5 mL final volume, 20 hour incubation time) with the "Heavy" reaction containing NEDDylator(10 $\mathrm{K}$ )-DS1 plus DMSO and the "Light" reaction containing NEDDylator(10 $\Delta \mathrm{K}$ )-DS1 plus $10 \mu \mathrm{M}$ of dasatinib (Figure S8). After incubation, the lysate was denatured by addition of urea to a final concentration of $8 \mathrm{M}$. The "Heavy" and "Light" reactions where then combined, and the mixture was incubated with $500 \mu \mathrm{L}$ of settled Pierce $^{\mathrm{TM}}$ High Capacity NeutrAvidin ${ }^{\mathrm{TM}}$ Agarose resin overnight $(\sim 16 \mathrm{hr})$ at room temperature. The resin was washed $2 \mathrm{x}$ with wash buffer 1 (50 mM Tris, $\mathrm{pH} 8.0,200 \mathrm{mM} \mathrm{NaCl}, 0.2 \%$ SDS, $8 \mathrm{M}$ Urea), $1 \mathrm{x}$ with wash buffer 2 (50 mM Tris, $\mathrm{pH} 8.0,200 \mathrm{mM} \mathrm{NaCl}, 2 \%$ SDS, 8M Urea), $1 \mathrm{x}$ with wash buffer $3(50 \mathrm{mM}$ Tris, $\mathrm{pH} 8.0,200 \mathrm{mM} \mathrm{NaCl}, 8 \mathrm{M}$ Urea), $2 \mathrm{x}$ with wash buffer 4 (50 $\mathrm{mM}$ Tris, $\mathrm{pH}$ 8.0, $0.5 \mathrm{mM}$ EDTA, $1 \mathrm{mM}$ DTT), and $2 \mathrm{x}$ with wash buffer 5 (50 $\mathrm{mM}$ ammonium bicarbonate, $\mathrm{pH}$ 7.8). Resin bound proteins were subjected to on-bead reduction (10 mM DTT, $1 \mathrm{hr}$ room temperature incubation), alkylation (40 $\mathrm{mM}$ IAM, $1 \mathrm{hr}$ room temperature incubation in the dark) and trypsinization (10 $\mu \mathrm{g}$ sequencing grade modified trypsin (Promega), $37^{\circ} \mathrm{C}$ incubation overnight). Eluted peptides were desalted with a $\mathrm{C}_{18}$ Ziptip (Millipore) following the manufacture's recommended protocol. Peptides were dried in vacuo and placed at $-80{ }^{\circ} \mathrm{C}$ until analyzed by liquid chromatography-tandem mass spectrometry. This protocol was performed twice, as two independent replicates.

\section{Mass Spectrometry and Data Analysis}

Liquid chromatography-tandem mass spectrometry (LC-MS/MS) was carried out on a reverse phase LC interfaced with a Q Exactive Plus (ThermoFisher Scientific) hybrid mass spectrometer. The nanoflow HPLC (Dionex UltiMate 3000, ThermoFisher Scientific) was equipped with an analytical column (Acclaim PepMap RSLC, $75 \mu \mathrm{m}$ X $150 \mathrm{~mm}$, ThermoFisher Scientific). Each replicate was injected 4 times. For the first two injections, peptides were eluted over a linear gradient from $97 \%$ solvent A $(0.1 \%$ formic acid in water) to $40 \%$ solvent B $(0.1 \%$ formic acid in acetonitrile) at $300 \mathrm{~nL} / \mathrm{min}$ over 125 minutes. For the second two injections, peptides were eluted over a linear gradient from $97 \%$ solvent A $(0.1 \%$ formic acid in water) to 
$40 \%$ solvent $\mathrm{B}(0.1 \%$ formic acid in acetonitrile $)$ at $300 \mathrm{~nL} / \mathrm{min}$ over 225 minutes. For all injections, MS and MS/MS spectra were acquired in a data-dependent mode with MS spectra acquired in the Orbitrap at 70,000 resolving power and $10 \mathrm{MS} / \mathrm{MS}$ spectra acquired per MS. Dynamic exclusion was set at 20 seconds to prevent repeated MS/MS on the same precursor ion.

For each injection of each replicate, peptide sequences were assigned using the ProteinProspector database search engine v 5.13.2 (http://prospector.ucsf.edu) against the SwissProt human protein database (2015.3.5) supplemented with the sequences of HB-NEDD8 and NEDDylator $(10 \Delta \mathrm{K})$. Search parameters included a precursor mass tolerance of $6 \mathrm{ppm}$, fragment ion mass tolerance of $20 \mathrm{ppm}$, up to 3 missed trypsin cleavages, constant carbamidomethylation of Cys, variable modifications of Acetylation (Protein N-term), Acetylation+Oxidation (Protein N-term M), Gln->pyro-Glu (N-term Q), GlyGly (Uncleaved K), Met-loss (Protein N-term M), Met-loss+Acetylation (Protein N-term M), and Oxidation (M). To identify "Heavy" peptides a separate search was performed using the same parameters with the addition of constant a constant ${ }^{13} \mathrm{C}_{6}{ }^{15} \mathrm{~N}_{2}$ Lys and ${ }^{13} \mathrm{C}_{6}{ }^{15} \mathrm{~N}_{4}$ Arg modification. The MS/MS spectra were also searched against a random decoy protein database for evaluating the false positive rates. At the maximum expectation value of peptide set at 0.0005 , the number of decoy peptides identified was $0.2 \%$ for Replicate 1 and $0.19 \%$ for Replicate 2. All peptides identified from the four injections of Replicate 1 were combined as a union and the area of their "Light" and "Heavy" MS1 peaks were quantified using ProteinProspector to give a single data set for Replicate 1 (Table S1Tab1). All Replicate 2 injections were similarly combined (Table S1-Tab2). Each of these datasets was then curated to remove decoy peptides, HB-NEDD8 peptides, NEDDylator(10 $\Delta \mathrm{K})$ peptides, NEDD8-E1 peptides, and keratin peptides, as these would interfere with analysis of the Heavy/Light SILAC ratios. Additionally, any peptides that showed a cosine similarity score (cs) of less then 0.7 for either MS1 peak were removed, as this data was deemed unreliable for Heavy/Light SILAC ratio quantification. These curated datasets (Table S1-Tab3-4) were analyzed using RStudio (https://www.rstudio.com/). Protein median Heavy/Light SILAC ratios were only calculated and plotted for proteins that had more than one unique peptide, and were observed in both Replicate 1 and 2 data sets. The protein assignments given by ProteinProspector were used for plotting, and homologous proteins were not taken into account for this analysis. Further assignment of peptides from enriched dasatinib targets to homologous proteins was performed using ProteinProspector (Table S1-Tab5-6).

\section{Supplemental References}

(1) Karaman, M. W.; Herrgard, S.; Treiber, D. K.; Gallant, P.; Atteridge, C. E.; Campbell, B. T.; Chan, K. W.; Ciceri, P.; Davis, M. I.; Edeen, P. T.; Faraoni, R.; Floyd, M.; Hunt, J. P.; Lockhart, D. J.; Milanov, Z. V.; Morrison, M. J.; Pallares, G.; Patel, H. K.; Pritchard, S.; Wodicka, L. M.; Zarrinkar, P. P. Nat. Biotechnol. 2008, 26, 127.

(2) Krishnamurty, R.; Brigham, J. L.; Leonard, S. E.; Ranjitkar, P.; Larson, E. T.; Dale, E. J.; Merritt, E. A.; Maly, D. J. Nat. Chem. Biol. 2013, 9, 43.

(3) Hill, Z. B.; Perera, B. G.; Andrews, S. S.; Maly, D. J. ACS Chem. Biol. 2012, 7, 487.

(4) Lemercier, G.; Gendreizig, S.; Kindermann, M.; Johnsson, K. Angew. Chem. Int. Ed. Engl. 2007, 46, 4281-4.

(5) Zhuang, M.; Guan, S.; Wang, H.; Burlingame, A. L.; Wells, J. A. Mol. Cell 2013, 49, 273.

(6) Seeliger, M. A.; Young, M.; Henderson, M. N.; Pellicena, P.; King, D. S.; Falick, A. M.; Kuriyan, J. Protein Sci. 2005, 14, 3135. 
(7) Seiler, C. Y.; Park, J. G.; Sharma, A.; Hunter, P.; Surapaneni, P.; Sedillo, C.; Field, J.; Algar, R.; Price, A.; Steel, J.; Throop, A.; Fiacco, M.; LaBaer, J. Nucleic Acids Res. 2014, 42, D1253. 